\title{
New N-bridgehead heterocyclic compounds. I. Carbamoyl-substituted indolizines and benzoindolizines
}

\author{
Emilian Georgescu, ${ }^{\text {a }}$ Florentina Georgescu, ${ }^{\text {a }}$ Mariana G. Danila, ${ }^{\text {b }}$ Petru I. Filip, \\ Constantin Drăghici, ${ }^{b}$ and Miron T. Căproiu ${ }^{b}$ \\ ${ }^{a}$ S.C. OLTCHIM - Research Center, 1 Uzinei St., Ramnicu Valcea 1000, Romania \\ ${ }^{b}$ Romanian Academy - Centre of Organic Chemistry, 202B Independentei Spl., Bucharest 71141, \\ Romania \\ E-mail:florentinafg@yahoo.com;sinteze@oltchim.onix.ro
}

In commemoration of the $100^{\text {th }}$ birthday of Prof. C. D. Nenitzescu

(received 23 Aug 2001; accepted 25 Nov 2001; published on the web 03 Dec 2001)

\begin{abstract}
Quaternary salts obtained by the reaction of several pyridines and benzopyridines with chloro- or bromoacetanilides were reacted with corresponding activated alkynes in the presence of an oxirane, yielding new carbamoyl-substituted indolizines and benzoindolizines derivatives. Other new 3-carbamoyl substituted indolizine and pyrrolo[2,1-a]isoquinoline derivatives were obtained by heating the intermediate N-metylcarbamoyl quaternary salts, in the presence of an acid acceptor, with alkenes and tetrapyridinecobalt(II)dichromate as a reaction promoter and dehydrogenating catalyst. The new compounds are fully characterised by elemental microanalysis and IR, ${ }^{1} \mathrm{H}$ and ${ }^{13} \mathrm{C}$ NMR spectra.
\end{abstract}

Keywords: Haloacetanilides as quaternizing agents, 1,3-dipolar cycloaddition reaction, carbamoylmethylide, 3-carbamoyl-indolizines, 1-carbamoylpyrrolo[1,2-a]quinoline, 3carbamoylpyrrolo[2,1-a]isoquinoline

\section{Introduction}

The indolizines have been subject of considerable interest from physical, chemical and biological points of view. ${ }^{1,2}$ The presence of a carbamoyl group on the pyrrole ring of the indolizines should have interesting effects on their chemical and biological properties. One of the most important methods for the synthesis of indolizines and benzoindolizines derivatives is based on 1,3-dipolar cycloaddition reactions of $\mathrm{N}$-heterocyclic ylides with electron-deficient alkynes or alkenes. ${ }^{3-5}$ The N-heterocyclic ylides could be obtained by the dehydrohalogenation of the corresponding quaternary salts of $\mathrm{N}$-heterocyclic compounds. ${ }^{4,5}$

Herein we report new carbamoyl-substituted N-bridgehead heterocyclic compounds obtained by the reactions of $\mathrm{N}$-heterocyclic compounds with chloroacetanilides or bromoacetanilides 
followed by the direct reactions of the intermediate N-methylcarbamoyl quaternary salts with activated alkynes or alkenes.

\section{Results and Discussion}

\section{N-Methylcarbamoyl quaternary salts}

By the quaternisation reactions of several pyridine, quinoline and isoquinoline derivatives with chloro- or bromoacetanilides the intermediate N-methylcarbamoyl quaternary salts 1-3 appeared easily accessible (Scheme 1, Table 1). The structures of the quaternary salts $\mathbf{1 - 3}$ were confirmed by chemical and spectral analyses. Some of the quaternary salts couldn't be separated and purified. They were used as crude products in the next step.

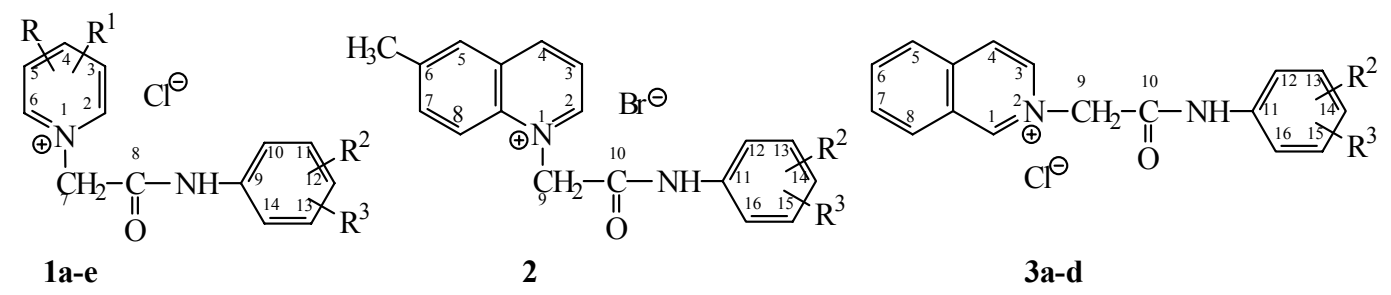

\section{Scheme 1}

Table 1. N-Methylcarbamoyl quaternary salts 1-3

\begin{tabular}{ccccccc}
\hline Compound & $\mathrm{R}$ & $\mathrm{R}^{1}$ & $\mathrm{R}^{2}$ & $\mathrm{R}^{3}$ & m.p. $\left({ }^{\circ} \mathrm{C}\right)$ & yield (\%) \\
\hline $\mathbf{1 a}$ & $\mathrm{H}$ & $\mathrm{H}$ & $2-\mathrm{F}$ & $\mathrm{H}$ & $238-240$ & 83 \\
$\mathbf{1 b}$ & $\mathrm{H}$ & $\mathrm{H}$ & $3-\mathrm{CF}_{3}$ & $\mathrm{H}$ & $211-213$ & 81 \\
$\mathbf{1 c}$ & $4-\mathrm{CH}_{3}$ & $\mathrm{H}$ & $3-\mathrm{CF}_{3}$ & $\mathrm{H}$ & $222-225$ & 76 \\
$\mathbf{1 d}$ & $4-\mathrm{CH}_{3}$ & $\mathrm{H}$ & $2-\mathrm{F}$ & $\mathrm{H}$ & $246-249$ & 84 \\
$\mathbf{1 e}$ & $2-\mathrm{CH}_{3}$ & $5-\mathrm{C}_{2} \mathrm{H}_{5}$ & $2-\mathrm{C}_{2} \mathrm{H}_{5}$ & $6-\mathrm{C}_{2} \mathrm{H}_{5}$ & $208-210$ & 94 \\
$\mathbf{2}$ & $6-\mathrm{CH}_{3}$ & $\mathrm{H}$ & $3-\mathrm{CF}_{3}$ & $\mathrm{H}$ & $228-230$ & 96 \\
$\mathbf{3 a}$ & - & $\mathrm{H}$ & $4-\mathrm{CH}_{3}$ & $\mathrm{H}$ & $136-139$ & 82 \\
$\mathbf{3 b}$ & - & $\mathrm{H}$ & $2-\mathrm{F}$ & $\mathrm{H}$ & $186-187$ & 76 \\
$\mathbf{3 c}$ & - & $\mathrm{H}$ & $2-\mathrm{OCH}_{3}$ & $\mathrm{H}$ & $247-250$ & 80 \\
$\mathbf{3 d}$ & - & $\mathrm{H}$ & $3-\mathrm{CF}_{3}$ & $\mathrm{H}$ & $252-255$ & 76 \\
\hline
\end{tabular}

\section{Carbamoyl-substituted indolizines and benzoindolizines}

By the direct reaction of the intermediate N-methylcarbamoyl pyridinium salts 1 with activated alkynes in an epoxide, as acid acceptor and reaction solvent, new indolizines bearing a carbamoyl group on the pyrrolo ring 5-12 were obtained (Scheme 2, Table 2). 

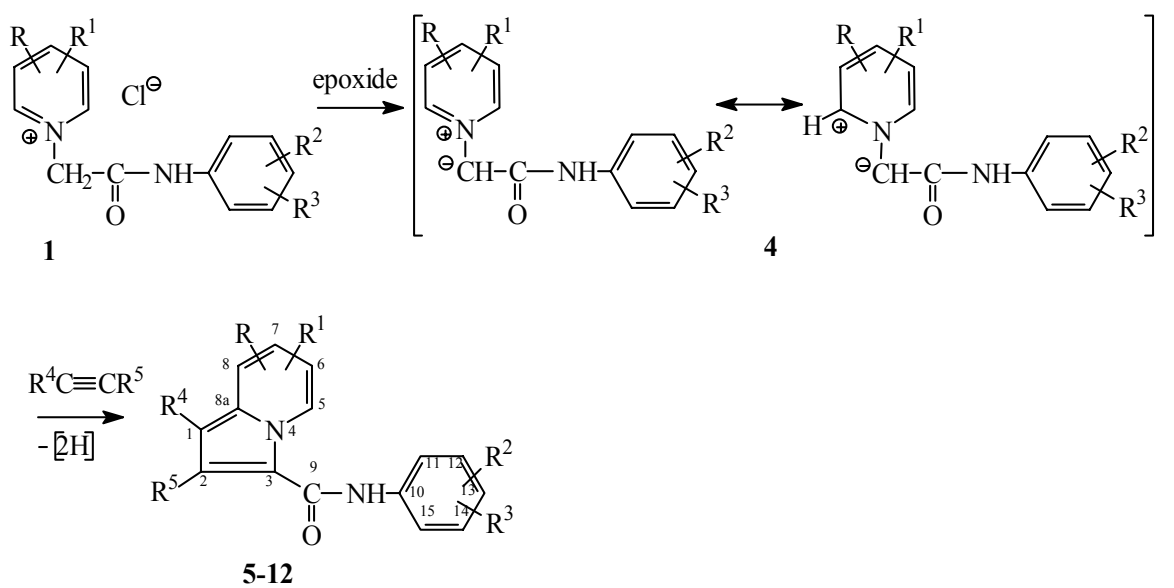

Scheme 2

Table 2. New prepared 3-carbamoylindolizines

\begin{tabular}{cccccccc}
\hline Compound & $\mathrm{R}$ & $\mathrm{R}^{1}$ & $\mathrm{R}^{2}$ & $\mathrm{R}^{3}$ & $\mathrm{R}^{4}$ & m.p. $\left({ }^{\circ} \mathrm{C}\right)$ & yield (\%) \\
\hline $\mathbf{5}$ & $\mathrm{H}$ & $\mathrm{H}$ & $2-\mathrm{F}$ & $\mathrm{H}$ & $\mathrm{CO}_{2} \mathrm{C}_{2} \mathrm{H}_{5}$ & $160-162$ & 44 \\
$\mathbf{6}$ & $\mathrm{H}$ & $\mathrm{H}$ & $2-\mathrm{C}_{2} \mathrm{H}_{5}$ & $\mathrm{H}$ & $\mathrm{CO}_{2} \mathrm{C}_{2} \mathrm{H}_{5}$ & $172.5-174$ & 51 \\
$\mathbf{7}$ & $\mathrm{H}$ & $\mathrm{H}$ & $3-\mathrm{CF}_{3}$ & $\mathrm{H}$ & $\mathrm{CO}_{2} \mathrm{C}_{2} \mathrm{H}_{5}$ & $171-172$ & 41 \\
$\mathbf{8}$ & $7-\mathrm{CH}_{3}$ & $\mathrm{H}$ & $2-\mathrm{F}$ & $\mathrm{H}$ & $\mathrm{CO}_{2} \mathrm{C}_{2} \mathrm{H}_{5}$ & $153-154$ & 42 \\
$\mathbf{9}$ & $7-\mathrm{CH}_{3}$ & $\mathrm{H}$ & $3-\mathrm{CF}_{3}$ & $\mathrm{H}$ & $\mathrm{CO}_{2} \mathrm{C}_{2} \mathrm{H}_{5}$ & $183-185$ & 39 \\
$\mathbf{1 0}$ & $7-\mathrm{CH}_{3}$ & $\mathrm{H}$ & $2-\mathrm{CH}_{3}$ & $6-\mathrm{C}_{2} \mathrm{H}_{5}$ & $\mathrm{COC}_{6} \mathrm{H}_{5}$ & $220-222$ & 28 \\
$\mathbf{1 1}$ & $5-\mathrm{CH}_{3}$ & $8-\mathrm{C}_{2} \mathrm{H}_{5}$ & $2-\mathrm{C}_{2} \mathrm{H}_{5}$ & $6-\mathrm{C}_{2} \mathrm{H}_{5}$ & $\mathrm{COCH}_{3}$ & $207-210$ & 26 \\
$\mathbf{1 2}$ & $5-\mathrm{CH}_{3}$ & $8-\mathrm{C}_{2} \mathrm{H}_{5}$ & $2-\mathrm{C}_{2} \mathrm{H}_{5}$ & $6-\mathrm{C}_{2} \mathrm{H}_{5}$ & $\mathrm{CO}_{2} \mathrm{CH}_{3}$ & $190-192$ & 50 \\
\hline
\end{tabular}

The structures of 3-carbamoylindolizines 5-12 were confirmed by microanalysis, IR, ${ }^{1} \mathrm{H}$ - and ${ }^{13} \mathrm{C}-\mathrm{NMR}$ spectral data.

The ${ }^{1} \mathrm{H}-\mathrm{NMR}$ spectra of 5-12 in $\mathrm{CDCl}_{3}$ reveal characteristic signals in the range $\delta$ 7.47-8.16 $(\mathrm{NH})$ and $\delta$ 7.78-8.16 (H-2). The ${ }^{1} \mathrm{H}-\mathrm{NMR}$ spectra of 5-9 show the signals for the ethyl protons of the carbethoxy group at $\delta$ 4.37-4.40 (q) and $\delta$ 1.41-1.44 (t). The ${ }^{1} \mathrm{H}-\mathrm{NMR}$ spectra of 10 and 12 exhibit the signals for the methyl protons of the acetyl and carbomethoxy group at $\delta 2.57$, respectively $\delta 3.90$.

The ${ }^{13} \mathrm{C}$-NMR spectra of 5-12 in $\mathrm{CDCl}_{3}$ show characteristic signals for the carbonyl carbon at $\delta 159-161$ (carbamoyl group), $\delta \sim 164$ (carboethoxy or carbomethoxy group) and $\delta 192.6$ (acetyl group), respectively.

Based on this one-pot procedure new 1-[(3-trifluoromethylphenyl)carbamoyl]-7methylpyrrolo[1,2-a]quinoline 13 and 3-carbamoyl substituted pyrrolo[2,1-a]-isoquinolines 1429 were obtained (Scheme 3, Table 3). 

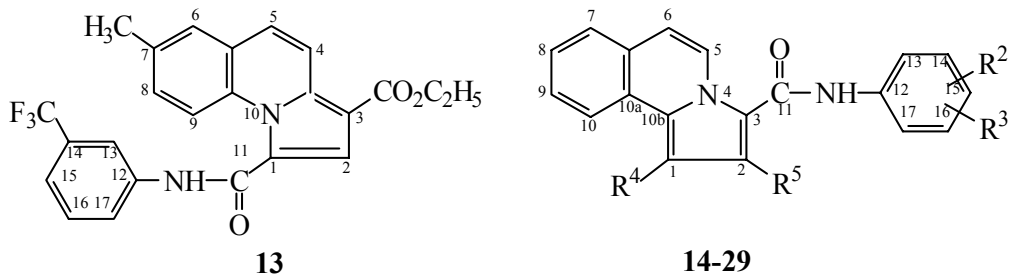

\section{Scheme 3}

The structures of new carbamoyl-substituted benzoindolizines 13 and 14-29 were confirmed by microanalysis, IR, ${ }^{1} \mathrm{H}$ - and ${ }^{13} \mathrm{C}-\mathrm{NMR}$ analysis.

For example, the IR spectra of 13-29 exhibit the characteristic absorption bands at about $3300 \mathrm{~cm}^{-1}$ and $3100 \mathrm{~cm}^{-1}(\mathrm{NH})$ and characteristic $\mathrm{C}=\mathrm{O}$ absorption bands at about $1700 \mathrm{~cm}^{-1}$ (COOMe/COOEt) and $1660 \mathrm{~cm}^{-1}\left(\mathrm{C}=\mathrm{O}\right.$ from carbamoyl group). The ${ }^{1} \mathrm{H}-\mathrm{NMR}$ spectra of 13-29, in $\mathrm{CDCl}_{3}$, present signals at $\delta 7.86-8.39(\mathrm{NH})$ in $\mathrm{CDCl}_{3}$, respectively at $\delta 10.21-11.12(\mathrm{NH})$ in a mixture of $\mathrm{CDCl}_{3}$ and trifluoroacetic acid, and two doublets at $\delta$ 7.02-7.20 and $\delta$ 9.29-9.47 $(\mathrm{J}$ 7.5-7.8 Hz). These latter signals were attributed to the protons $\mathrm{H}-4$ and $\mathrm{H}-5$ (in 13), respectively H-6 and H-5 (in 14-29). In the ${ }^{1} \mathrm{H}-\mathrm{NMR}$ spectra of 14-18 and 22 the signal at $\delta$ 7.73-7.83 was attributed to the H-2 proton. The methyl, respectively ethyl, signals from ester groups appears at $\delta 3.97-4.03$, respectively $\delta 4.40(\mathrm{q})$ and $\delta 1.46(\mathrm{t})$.

Table 3. New 3-carbamoyl pyrrolo[2,1-a]isoquinolines 14-29

\begin{tabular}{ccccccc}
\hline Compound & $\mathrm{R}^{2}$ & $\mathrm{R}^{3}$ & $\mathrm{R}^{4}$ & $\mathrm{R}^{5}$ & m.p. $\left({ }^{\circ} \mathrm{C}\right)$ & yield (\%) \\
\hline $\mathbf{1 4}$ & $2-\mathrm{F}$ & $\mathrm{H}$ & $\mathrm{CO}_{2} \mathrm{C}_{2} \mathrm{H}_{5}$ & $\mathrm{H}$ & $152-154$ & 53 \\
$\mathbf{1 5}$ & $3-\mathrm{CH}_{3}$ & $\mathrm{H}$ & $\mathrm{CO}_{2} \mathrm{CH}_{3}$ & $\mathrm{H}$ & $166-168$ & 51 \\
$\mathbf{1 6}$ & $2-\mathrm{OCH}_{3}$ & $\mathrm{H}$ & $\mathrm{CO}_{2} \mathrm{C}_{2} \mathrm{H}_{5}$ & $\mathrm{H}$ & $164-166$ & 50 \\
$\mathbf{1 7}$ & $3-\mathrm{OCH}_{3}$ & $\mathrm{H}$ & $\mathrm{CO}_{2} \mathrm{C}_{2} \mathrm{H}_{5}$ & $\mathrm{H}$ & $171-172$ & 54 \\
$\mathbf{1 8}$ & $3-\mathrm{CF}_{3}$ & $\mathrm{H}$ & $\mathrm{CO}_{2} \mathrm{C}_{2} \mathrm{H}_{5}$ & $\mathrm{H}$ & $201-203$ & 61 \\
$\mathbf{1 9}$ & $2-\mathrm{CH}_{3}$ & $\mathrm{H}$ & $\mathrm{CO}_{2} \mathrm{CH}_{3}$ & $\mathrm{CO}_{2} \mathrm{CH}_{3}$ & $152-154$ & 46 \\
$\mathbf{2 0}$ & $4-\mathrm{CH}_{3}$ & $\mathrm{H}$ & $\mathrm{CO}_{2} \mathrm{CH}_{3}$ & $\mathrm{CO}_{2} \mathrm{CH}_{3}$ & $166-167.5$ & 44 \\
$\mathbf{2 1}$ & $3-\mathrm{CF}_{3}$ & $\mathrm{H}$ & $\mathrm{CO}_{2} \mathrm{CH}_{3}$ & $\mathrm{CO}_{2} \mathrm{CH}_{3}$ & $143-145$ & 44 \\
$\mathbf{2 2}$ & $3-\mathrm{Cl}_{2}$ & $4-\mathrm{F}$ & $\mathrm{CO}_{2} \mathrm{C}_{2} \mathrm{H}_{5}$ & $\mathrm{H}$ & $202-204$ & 43 \\
$\mathbf{2 3}$ & $4-\mathrm{Cl}_{2}$ & $\mathrm{H}$ & $\mathrm{CO}_{2} \mathrm{CH}_{3}$ & $\mathrm{CO}_{2} \mathrm{CH}_{3}$ & $177-179$ & 29 \\
$\mathbf{2 4}$ & $2-\mathrm{CH}_{3}$ & $6-\mathrm{CH}$ & $\mathrm{CO}_{2} \mathrm{CH}_{3}$ & $\mathrm{CO}_{2} \mathrm{CH}_{3}$ & $180-182$ & 34 \\
$\mathbf{2 5}$ & $2-\mathrm{C}_{2} \mathrm{H}_{5}$ & $6-\mathrm{C}_{2} \mathrm{H}_{5}$ & $\mathrm{CO}_{2} \mathrm{CH}_{3}$ & $\mathrm{CO}_{2} \mathrm{CH}_{3}$ & $160-162$ & 38 \\
$\mathbf{2 6}$ & $2-\mathrm{CH}_{3}$ & $3-\mathrm{Cl}$ & $\mathrm{CO}_{2} \mathrm{CH}_{3}$ & $\mathrm{CO}_{2} \mathrm{CH}_{3}$ & $167-169$ & 39 \\
$\mathbf{2 7}$ & $2-\mathrm{CH}_{3}$ & $4-\mathrm{Cl}$ & $\mathrm{CO}_{2} \mathrm{CH}_{3}$ & $\mathrm{CO}_{2} \mathrm{CH}_{3}$ & $171-172$ & 41 \\
$\mathbf{2 8}$ & $2-\mathrm{CH}_{3}$ & $5-\mathrm{Cl}$ & $\mathrm{CO}_{2} \mathrm{CH}_{3}$ & $\mathrm{CO}_{2} \mathrm{CH}_{3}$ & $198-200$ & 36 \\
$\mathbf{2 9}$ & $3,4-\mathrm{methylenedioxy}$ & $\mathrm{CO}_{2} \mathrm{CH}_{3}$ & $\mathrm{CO}_{2} \mathrm{CH}_{3}$ & $185-186$ & 57 \\
\hline
\end{tabular}

All the ${ }^{13} \mathrm{C}-\mathrm{NMR}$ spectra of 13-29 reveal characteristic signals for the carbonyl carbon at $\delta \sim 160$ (carbamoyl group) and $\delta \sim 164$ (carboethoxy or carbomethoxy group). 
Propenoxide was used as acid acceptor in all these reactions, and the activated alkynes were methyl or ethyl propiolate, 1-butyne-3-one, phenylethynyl ketone or dimethylacetylene dicarboxylate.

In the regular conditions of a 1,3-dipolar cycloaddition reaction, $\mathrm{N}$-heterocyclic ylides react slowly even with strong activated alkenes. ${ }^{4,5}$ In some cases aromatic indolizines were prepared by two-steps procedures, in which the initially formed tetrahydroindolizines or dihydroindolizines are dehydrogenated by treatment with suitable reagents. ${ }^{6-10}$

By 1,3-dipolar cycloaddition reactions of the 4-methylpyridinium-, respectively isoquinolinium-carbamoylmethylides, generated in situ from the corresponding quaternary salts, with acrylonitrile and respectively with crotononitrile, in the presence of tetrapyridinecobalt(II)dichromate, ${ }^{11,12}$ as a reaction promoter and dehydrogenating catalyst, other new 3-carbamoyl substituted indolizine 30, respectively pyrrolo[2,1-a]-isoquinolines 31-32 (Scheme 4, Table 4) were obtained.

These cycloadition reactions could be accomplished by treating the corresponding quaternary salts with alkenes and tetrapyridinecobalt(II)dichromate, in $\mathrm{DMF}$ at $90^{\circ} \mathrm{C}$, using pyridine as hydrobromic acid acceptor, ${ }^{11,12}$ or by heating the N-methylcarbamoyl quaternary salts with alkenes and tetrapyridinecobalt(II)dichromate in 1,2-epoxybutane used as acid acceptor and solvent. ${ }^{13}$

The structures of new carbamoyl-substituted indolizine $\mathbf{3 0}$ and pyrrolo[2,1-a]-isoquinolines 31-32 were confirmed by IR, ${ }^{1} \mathrm{H}$ - and ${ }^{13} \mathrm{C}-\mathrm{NMR}$ analysis.

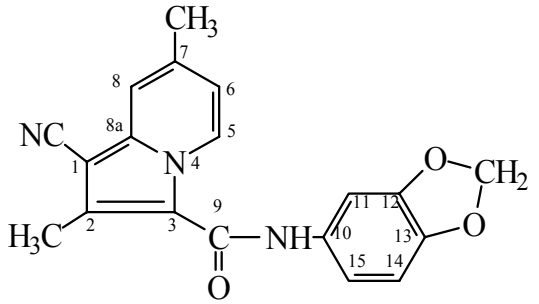

30

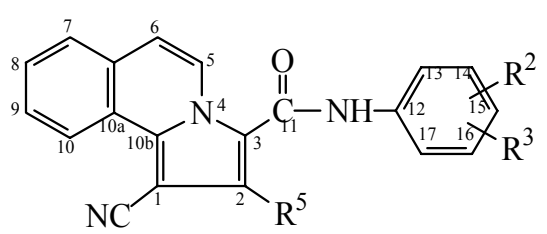

31-32

\section{Scheme 4}

Table 4. New 3-carbamoyl indolizine and benzoindolizine derivatives 30-32

\begin{tabular}{|c|c|c|c|c|c|c|}
\hline Compound & $\mathrm{R}^{1}$ & $\mathrm{R}^{2}$ & $\mathrm{R}^{3}$ & $\mathrm{R}^{5}$ & m.p. $\left({ }^{\circ} \mathrm{C}\right)$ & yield $(\%)$ \\
\hline 30 & 7- $\mathrm{CH}_{3}$ & \multicolumn{2}{|c|}{ 3,4-methylenedioxy } & $\mathrm{CH}_{3}$ & $246-246.5$ & 12.0 \\
\hline 31 & $\mathrm{H}$ & $3-\mathrm{CF}_{3}$ & $\mathrm{H}$ & $\mathrm{CH}_{3}$ & $225-228$ & 34 \\
\hline 32 & $\mathrm{H}$ & $3-\mathrm{OCH}_{3}$ & $\mathrm{H}$ & $\mathrm{H}$ & $286-289$ & 38 \\
\hline
\end{tabular}

The IR spectra of 30-32 exhibit the single $\mathrm{CN}$ absorbtion bands at $\left.2204 \mathrm{~cm}^{-1} \mathbf{( 3 0 ,}, \mathbf{3 1}\right)$ or $2213 \mathrm{~cm}^{-1}$ (32), two NH absorption bands at about $3300 \mathrm{~cm}^{-1}$ and $3100 \mathrm{~cm}^{-1}$, characteristic $\mathrm{C}=\mathrm{O}$ absorption bands at 1636-1669 $\mathrm{cm}^{-1}$ and 1536-1547 $\mathrm{cm}^{-1}$. The ${ }^{1} \mathrm{H}-\mathrm{NMR}$ spectrum of 30 in a mixture of $\mathrm{CDCl}_{3}$ and trifluoroacetic acid reveals the signals at $\delta 7.82(\mathrm{NH})$ and $\delta 7.40(\mathrm{H}-8)$, as a broad singlet, a doublet at $\delta 9.09(J 7.2 \mathrm{~Hz})$ attributed to $\mathrm{H}-5$ and a double doublet at $\delta 6.82(J$ 
7.2 and $1.8 \mathrm{~Hz})$ attributed to $\mathrm{H}-6$. The characteristic two methyl signals appear at $\delta 2.45\left(2-\mathrm{CH}_{3}\right)$ and $2.69\left(7-\mathrm{CH}_{3}\right)$ and the methylene protons appear at $\delta 6.00$. The ${ }^{1} \mathrm{H}-\mathrm{NMR}$ spectra of $\mathbf{3 1}$ (in $\mathrm{CDCl}_{3}$ ), respectively of $\mathbf{3 2}$ (in a mixture of $\mathrm{CDCl}_{3}$ and trifluoroacetic acid), show the characteristic NH signals at $\delta 8.11$, respectively $\delta 9.30$. The two doublets at $\delta 8.71$ and $\delta 6.82$ (for 31), and respectively at $\delta 9,16$ and $\delta 7.23$ (for 32), were attributed to H-5 and H-6. The characteristic signals for methyl protons appeared at $\delta 2.75$, respectively $\delta 3.88$. The ${ }^{13} \mathrm{C}$-NMR spectra of 30-32 exhibit the characteristic signals for the $\mathrm{C}=\mathrm{O}$ carbon at $\delta \sim 160$ (carbamoyl group) and for the $\mathrm{C} \equiv \mathrm{N}$ carbon at $\delta \sim 116$.

In conclusion, the otherwise not easily accessible indolizines and benzoindolizines bearing carbamoyl groups on the pyrrolo ring are readily prepared by the simple one-pot synthesis described herein.

\section{Experimental Section}

General Procedures. Melting points were determined on a Boetius apparatus and are uncorrected. The IR spectra were recorded on a Nicolet Impact 410 spectrometer, in $\mathrm{KBr}$ pellets. The ${ }^{1} \mathrm{H}$ - and ${ }^{13} \mathrm{C}-\mathrm{NMR}$ spectra were registered with a Varian Gemini $300 \mathrm{BB}$ instrument at ambient temperature using TMS as internal standard; for unambiguous assignment ${ }^{1} \mathrm{H}$ decoupling COSY $\left({ }^{1} \mathrm{H}-{ }^{1} \mathrm{H}\right)$ and $\operatorname{COSY}\left({ }^{1} \mathrm{H}_{-}{ }^{13} \mathrm{C}\right)$ were used. The solvent used was $\mathrm{CDCl}_{3}$ for the compounds 5-12, 14-17, 19-25 and 31, or a mixture of 10:1 molar ratio $\mathrm{CDCl}_{3}$ :TFA only for the compounds 1a-1e, 2, 3a-3d, 13, 18, 26-30 and 32. Elemental analyses were carried out on a Carlo Erba 1106 Elemental Analyzer. Pyridine, quinoline and isoquinoline derivatives were commercially available products (Aldrich). Chloro- and bromoacetanilides were obtained from the corresponding aromatic amines and chloroacetyl chloride, respectively bromoacetyl bromide. Tetrapyridinecobalt(II)dichromate (TPCD) was prepared according to a previously described method. ${ }^{11}$

\section{N-Methylcarbamoyl quaternary salts. General procedure}

A mixture of a N-heterocyclic compound $(20 \mathrm{mmol})$ and the corresponding chloroacetanilide or bromoacetanilide $(20 \mathrm{mmol})$ in chloroform $(50 \mathrm{~mL})$ was heated at reflux for 20 hours. The mixture was cooled and left overnight at the room temperature. The solid product was filtered, washed with a mixture of methylene dichloride-diethyl ether $(30 \mathrm{~mL})$ and recrystallised from methanol or methanol/diethyl ether.

The yields and m. p. are shown in Table 1. The spectral data are given below.

1-[N-(2-Fluorophenyl)carbamoylmethyl]pyridinium chloride (1a). IR $\vee$ 3144, 3093, 1685, 1554. ${ }^{1} \mathrm{H}-\mathrm{NMR}(\delta \mathrm{ppm}, J \mathrm{~Hz}): 9.43(1 \mathrm{H}, \mathrm{s}, \mathrm{NH}) ; 8.89(2 \mathrm{H}, \mathrm{d}, 6.6,2-\mathrm{H}, 6-\mathrm{H}) ; 8.56(1 \mathrm{H}, \mathrm{t}, 7.6,4-$ $\mathrm{H}) ; 8.10(2 \mathrm{H}, \mathrm{dd}, 6.6,7.6,3-\mathrm{H}, 5-\mathrm{H},) ; 7.68(1 \mathrm{H}, \mathrm{td}, 8.8,1.80,14-\mathrm{H}) ; 7.08-7.23(3 \mathrm{H}, \mathrm{m}, 11-\mathrm{H}, 12-$ $\mathrm{H}, 13-\mathrm{H}) ; 5.91\left(2 \mathrm{H}, \mathrm{s}, \mathrm{CH}_{2}\right) .{ }^{13} \mathrm{C}-\mathrm{NMR} \delta 163.02(8-\mathrm{C}) ; 154.60$ (10-C, d, 248.1), $146.44(4-\mathrm{C})$; 146.01 (2-C, 6-C); 128.17 (3-C, 5-C, 14-C); 124.66 (12-C, d, 3.9); 124.32 (13-C); 122.95 (9-C, d, 11.1); 115.92 (11-C, d, 19.3); $62.83\left(\mathrm{CH}_{2}\right)$. Anal. calcd. for $\mathrm{C}_{13} \mathrm{H}_{12} \mathrm{ClFN}_{2} \mathrm{O}$ (266.70): C, 58.54; H, 4.53 ; N, 10.50\%. Found: C, 58.65; H, 4.59; N, 10.62\%. 
1-[N-(3-Trifluoromethylphenyl)carbamoylmethyl]pyridinium chloride (1b). IR $v$ 3246, 3085, 1707, 1566. ${ }^{1} \mathrm{H}-\mathrm{NMR}(\delta \mathrm{ppm}, J \mathrm{~Hz}): 10.28$ (1H, bs, NH), 8.90 (2H, dd, 6.6, 0.9, 2-H, 6-H); $8.49(1 \mathrm{H}, \mathrm{tt}, 7.8,0.9,4-\mathrm{H}) ; 8.03(2 \mathrm{H}, \mathrm{dd}, 7.8,6.6,3-\mathrm{H}, 5-\mathrm{H}) ; 7.90(1 \mathrm{H}, \mathrm{s}, 11-\mathrm{H}) ; 7.65(1 \mathrm{H}, \mathrm{m}$, $15-\mathrm{H}) ; 7.35-7.42(2 \mathrm{H}, \mathrm{m}, 13-\mathrm{H}, 14-\mathrm{H}) ; 5.88\left(2 \mathrm{H}, \mathrm{s}, \mathrm{CH}_{2}\right) .{ }^{13} \mathrm{C}-\mathrm{NMR} \delta 162.41$ (8-C); 146.08 (4C); 145.95 (2-C, 6-C); 137.16 (10-C); 131.40 (12-C, d, 33.1); 129.67 (14-C); 127.95 (3-C, 5-C); 123.53 (15-C); 122.00 (13-C); $121.95\left(\mathrm{CF}_{3}, \mathrm{q}, 272.2\right)$; 117.13 (11-C); $63.07\left(\mathrm{CH}_{2}\right)$. Anal. calcd. for $\mathrm{C}_{14} \mathrm{H}_{12} \mathrm{ClF}_{3} \mathrm{~N}_{2} \mathrm{O}$ (316.71): C, 53.09; H, 3.82; N, 8.85\%. Found: C, 53.19; H, 3.89; N, 8.93\%.

1-[N-(3-Trifluoromethylphenyl)carbamoylmethyl]-4-methylpyridinium chloride (1c). IR $\mathrm{v}$ 3252, 3031, 1693, 1574. ${ }^{1} \mathrm{H}-\mathrm{NMR}(\delta \mathrm{ppm}, J \mathrm{~Hz})$ : 9.95 (1H, bs, NH); 8.64 (2H, d, 6.7, 2-H, 6-H); $7.90(1 \mathrm{H}, \mathrm{bs}, 10-\mathrm{H}) ; 7.83(2 \mathrm{H}, \mathrm{d}, 6.7,3-\mathrm{H}, 5-\mathrm{H}) ; 7.48-7.62(3 \mathrm{H}, \mathrm{m}, 12-\mathrm{H}, 13-\mathrm{H}, 14-\mathrm{H}) ; 5.75(2 \mathrm{H}$, $\left.\mathrm{s}, \mathrm{CH}_{2}\right) ; 2.71\left(3 \mathrm{H}, \mathrm{s}, \mathrm{CH}_{3}\right) .{ }^{13} \mathrm{C}-\mathrm{NMR} \delta 163.40$ (8-C); 161.56 (4-C); 144.72 (2-C, 6-C); 136.32 (9-C); 131.69 (11-C, q, 33.1); 129.89 (14-C); 128.64 (3-C, 5-C); 124.06 (13-C); 123.53 (CF 3 , q, 272.3); 122.86 (12-C, q, 3.8); 117.76 (10-C, q, 4.1); $62.08\left(\mathrm{CH}_{2}\right) ; 22.19\left(\mathrm{CH}_{3}\right)$. Anal. calcd. for $\mathrm{C}_{15} \mathrm{H}_{14} \mathrm{ClF}_{3} \mathrm{~N}_{2} \mathrm{O}$ (330.73): C, 54.47; H, 4.26; N, 8.47\%. Found: C, 54.43; H, 4.21; N, 8.42\%.

1-[N-(2-Fluorophenyl)carbamoylmethyl]-4-methylpyridinium chloride (1d). IR $v 3162$, 3035, 1689, 1545. ${ }^{1} \mathrm{H}-\mathrm{NMR}(\delta \mathrm{ppm}, J \mathrm{~Hz}): 9.49$ (1H, s, NH); 8.68 (2H, d, 6.4, 2-H, 6-H); 7.78 $(2 \mathrm{H}, 6.4,3-\mathrm{H}, 5-\mathrm{H}) ; 7.74(1 \mathrm{H}, \mathrm{td}, 8.0,1.8,14-\mathrm{H}) ; 7.01-7.19(3 \mathrm{H}, \mathrm{m}, 11-\mathrm{H}, 12-\mathrm{H}, 13-\mathrm{H}) ; 5.83$ $\left(2 \mathrm{H}, \mathrm{s}, \mathrm{CH}_{2}\right) ; 2.66\left(3 \mathrm{H}, \mathrm{s}, \mathrm{CH}_{3}\right) .{ }^{13} \mathrm{C}-\mathrm{NMR} \delta 163.63(8-\mathrm{C}) ; 161.14$ (4-C); $154.30(10-\mathrm{C}, \mathrm{d}$, 247.8); 144.79 (2-C, 6-C); 128.47 (3-C, 5-C); 127.43 (14-C, d, 7.6); 124.49 (12-C, d, 3.7); 124.13 (13-C); 123.57 (9-C, d, 11.6); 115.67 (11-C, d, 19.1); $61.93\left(\mathrm{CH}_{2}\right) ; 22.04\left(\mathrm{CH}_{3}\right)$. Anal. calcd. for $\mathrm{C}_{14} \mathrm{H}_{14} \mathrm{ClFN}_{2} \mathrm{O}$ (280.72): C, 59.90; H, 5.03; N, 9.98\%. Found: C, 59.86; H, 5.12; N, 9.94\%.

1-[N-(2,6-Diethylphenyl)carbamoylmethyl]-2-ethyl-5-methylpyridinium chloride (1e). IR v 3392, 3049, 1673, 1540. ${ }^{1} \mathrm{H}-\mathrm{NMR}(\delta \mathrm{ppm}, J \mathrm{~Hz}): 10.93(1 \mathrm{H}, \mathrm{s}, \mathrm{NH}) ; 9.29(1 \mathrm{H}, \mathrm{d}, 1.90,6-\mathrm{H})$; 8.09 (1H, dd, 1.9, 8.1, 4-H); 7.70 (1H, d, 8.1, 3-H); 7.09-7.19 (3H, m, 11-H, 12-H, 13-H); 6.31 $\left(2 \mathrm{H}, \mathrm{s}, 7-\mathrm{CH}_{2}\right) ; 2.92\left(3 \mathrm{H}, \mathrm{s}, 2-\mathrm{CH}_{3}\right) ; 2.89\left(2 \mathrm{H}, \mathrm{q}, 6.9,5-\mathrm{CH}_{2}\right) ; 1.31\left(3 \mathrm{H}, \mathrm{t}, 6.9,5-\mathrm{C}_{2} \mathrm{H}_{5}\right) ; 2.66$ (4H, q, 2,6-diEt, 7.1); 1.14 (6H, t, 7.1, 2,6-diEt). ${ }^{13} \mathrm{C}-\mathrm{NMR} \delta 163.23$ (8-C); 153.20 (2-C); 146.19 (6-C); 144.61 (4-C); 141.12 (10-C); 142.12 (9-C); 141.12 (10-C, 14-C); 132.26 (5-C); 128.88 (3C); 127.69 (12-C); $125.88(11-\mathrm{C}, 13-\mathrm{C}) ; 60.03\left(7-\mathrm{CH}_{2}\right) ; 25.34\left(\mathrm{CH}_{2}\right.$ from 5-Et) $24.78\left(2 \mathrm{CH}_{2}\right.$ from 2,6-diEt); 20.37 (2- $\left.\mathrm{CH}_{3}\right) ; 14.37\left(2 \mathrm{CH}_{3}\right.$ from 2,6-diEt); $14.08\left(\mathrm{CH}_{3}\right.$ from 5-Et). Anal. calcd. for $\mathrm{C}_{20} \mathrm{H}_{27} \mathrm{ClN}_{2} \mathrm{O}$ (346.89): C, 69.25; H, 7.84; N, 8.07\%. Found: C, 69.53; H, 7.75; N, 8.05\%.

1-[N-(3-Trifluoromethylphenyl)carbamoylmethyl]-6-methylquinolinium bromide (2). IR v 3205, 3069, 1692, 1573. ${ }^{1} \mathrm{H}-\mathrm{NMR}(\delta \mathrm{ppm}, J \mathrm{~Hz}): 10.27(1 \mathrm{H}, \mathrm{s}, \mathrm{NH}) ; 9.15(1 \mathrm{H}, \mathrm{dd}, 6.0,1.5,2-\mathrm{H})$; $8.96(1 \mathrm{H}, \mathrm{bd}, 8.4,4-\mathrm{H}) ; 8.30(1 \mathrm{H}, \mathrm{d}, 9.1,8-\mathrm{H}) ; 8.10(1 \mathrm{H}, \mathrm{dd}, 9.1,20,7-\mathrm{H}) ; 8.06(1 \mathrm{H}, \mathrm{bs}, 5-\mathrm{H})$; $8.02(1 \mathrm{H}, \mathrm{dd}, 8.4,6.0,3-\mathrm{H}) ; 7.89(1 \mathrm{H}, \mathrm{bs}, 12-\mathrm{H}) ; 7.71(1 \mathrm{H}, \mathrm{m}, 15-\mathrm{H}) ; 7.47-7.51(2 \mathrm{H}, \mathrm{m}, 14-\mathrm{H}$, $16-\mathrm{H}) ; 6.33\left(2 \mathrm{H}, \mathrm{s}, \mathrm{CH}_{2}\right) ; 2.69\left(3 \mathrm{H}, \mathrm{s}, 6-\mathrm{CH}_{3}\right) .{ }^{13} \mathrm{C}-\mathrm{NMR} \delta 163.80(\mathrm{CO}) ; 148.94$ (2-C); 147.90 (4-C); 142.77 (8a-C); 139.88 (7-C); 137.66 (6-C); 136.23 (11-C); 131.91 (13-C, q, 33.1); 130.45 (4a-C); 129.98 (16-C); 129.40 (5-C); 124.24 (15-C); $123.56\left(\mathrm{CF}_{3}, \mathrm{q}, 272.2\right) ; 123.16$ (14-C, q, 3.5); 121.52 (3C); 117.98 (12-C, q, 3.6); $117.95(8-\mathrm{C}) ; 60.13\left(\mathrm{CH}_{2}\right) ; 21.23\left(\mathrm{CH}_{3}\right)$. Anal. calcd. for $\mathrm{C}_{19} \mathrm{H}_{16} \mathrm{BrF}_{3} \mathrm{~N}_{2} \mathrm{O}$ (425.24): C, 53.66; H, 3.79; N, 6.59\%. Found: C, 53.60; H, 3.85; N, 6.61\%.

2-[N-(4-Methylphenyl)carbamoylmethyl]isoquinolinium chloride (3a). IR $v$ 3235, 3049, 1636, 1544. ${ }^{1} \mathrm{H}-\mathrm{NMR}(\delta \mathrm{ppm}, J \mathrm{~Hz}): 9.79(1 \mathrm{H}, \mathrm{bs}, 1-\mathrm{H}) ; 9.67(1 \mathrm{H}, \mathrm{s}, \mathrm{NH}) ; 8.50(1 \mathrm{H}, \mathrm{d}, 6.7,3-$ 
$\mathrm{H}) ; 8.40(1 \mathrm{H}, \mathrm{d}, 8.4,8-\mathrm{H}) ; 8.31(1 \mathrm{H}, \mathrm{d}, 6.7,4-\mathrm{H}) ; 8.26(1 \mathrm{H}, \mathrm{ddd}, 1.1,6.7,8.4,6-\mathrm{H}) ; 8.20(1 \mathrm{H}$, $\mathrm{dd}, 1.1,8.3,5-\mathrm{H}) ; 8.07$ (1H, ddd, 1.2, 6.7, 8.3, 7-H); $7.33(2 \mathrm{H}, \mathrm{d}, 8.2,10-\mathrm{H}, 14-\mathrm{H}) ; 7.14(2 \mathrm{H}, \mathrm{d}$, 8.2, 11-H, 13-H); $5.90\left(2 \mathrm{H}, \mathrm{s}, \mathrm{CH}_{2}\right) ; 2.30\left(3 \mathrm{H}, \mathrm{s}, \mathrm{CH}_{3}\right) .{ }^{13} \mathrm{C}-\mathrm{NMR} \delta 162.96(\mathrm{CO}) ; 150.62(1-\mathrm{C})$; 137.96 (6-C); 137.84 (4a-C); 135.78 (12-C or C-9); 135.37 (3-C); 133.72 (9-C or 12-C); 131.79 (7-C); 130.72 (8-C); 127.49 (8a-C); 129.60 (11-C, 13-C); 127.22 (5-C); 126.02 (4-C); 120.97 (10-C, 14-C); $62.73\left(\mathrm{CH}_{2}\right) ; 20.66\left(\mathrm{CH}_{3}\right)$. Anal. calcd. for $\mathrm{C}_{18} \mathrm{H}_{17} \mathrm{ClN}_{2} \mathrm{O}$ (312.79): $\mathrm{C}, 69.12 ; \mathrm{H}$, $5.48 ;$ N, 8.95\%. Found: C, 69.20; H, 5.52; N, 8.59\%.

2-[N-(2-Fluorophenyl)carbamoylmethyl]isoquinolinium chloride (3b). IR $v$ 3184, 3047, 1702, 1548. ${ }^{1} \mathrm{H}-\mathrm{NMR}(\delta \mathrm{ppm}, J \mathrm{~Hz}): 9.76(1 \mathrm{H}, \mathrm{bs}, 1-\mathrm{H}) ; 9.66(1 \mathrm{H}, \mathrm{s}, \mathrm{NH}) ; 8.44(1 \mathrm{H}, \mathrm{dd}, 1.4,6.9$, $3-\mathrm{H}) ; 8.43(1 \mathrm{H}, \mathrm{dd}, 8.4,0.8,8-\mathrm{H}) ; 8.31(1 \mathrm{H}, \mathrm{d}, 6.9,4-\mathrm{H}) ; 8.24(1 \mathrm{H}, \mathrm{ddd}, 1.2,6.6,8.2,6-\mathrm{H}) ; 8.19$ $(1 \mathrm{H}, \mathrm{bd}, 8.2,5-\mathrm{H}) ; 8.05(1 \mathrm{H}, \mathrm{ddd}, 1.4,6.6,8.4,7-\mathrm{H}) ; 7.80(1 \mathrm{H}, \mathrm{td}, 7.7,1.9,14-\mathrm{H}) ; 7.07-7.22$ $(3 \mathrm{H}, \mathrm{m}, 11-\mathrm{H}, 12-\mathrm{H}, 13-\mathrm{H}) ; 6.06\left(2 \mathrm{H}, \mathrm{s}, \mathrm{CH}_{2}\right) .{ }^{13} \mathrm{C}-\mathrm{NMR} \delta 163.52(\mathrm{CO}) ; 154.38(10-\mathrm{C}, \mathrm{d}$, 248.5); 150.81 (1-C); 138.21 (6-C); 137.96 (4a-C); 135.49 (3-C); 132.01 (7-C); 130.90 (8-C); 127.62 (8a-C); 127.45 (12-C, d, 7.7); 127.29 (5-C); 126.05 (4-C); 124.52 (14-C, d, 3.9); 124.02 (13-C); 123.74 (9-C, d, 11.5); 115.83 (11-C, d, 19.0); $62.71\left(\mathrm{CH}_{2}\right)$. Anal. calcd. for $\mathrm{C}_{17} \mathrm{H}_{14} \mathrm{ClFN}_{2} \mathrm{O}$ (316.76): C, 64.46; H, 4.45; N, 8.84\%. Found: C, 64.52; H, 4.51; N, 8.88\%.

2-[N-(2-Methoxyphenyl)carbamoylmethyl]isoquinolinium chloride (3c). IR $v$ 3325, 3046, 1689, 1544. ${ }^{1} \mathrm{H}-\mathrm{NMR}(\delta \mathrm{ppm}, J \mathrm{~Hz}): 9.72(1 \mathrm{H}, \mathrm{bs}, \mathrm{H}-1) ; 9.31(1 \mathrm{H}, \mathrm{s}, \mathrm{NH}) ; 8.51(1 \mathrm{H}, \mathrm{dd}, 6.8,1.2$, $3-\mathrm{H})$; 8.39 (1H, bd, 8.4, 5-H); $8.28(1 \mathrm{H}, \mathrm{d}, 6.8,4-\mathrm{H}) ; 8.20(1 \mathrm{H}, \mathrm{ddd}, 1.1,7.2,8.4,6-\mathrm{H}) ; 8.15(1 \mathrm{H}$, bd, 7.2, 8-H); $8.00(1 \mathrm{H}$, ddd, 1.4, 7.2, 8.4, 7-H); $7.82(1 \mathrm{H}, \mathrm{ddd}, 7.9,7.6,1.5,14-\mathrm{H}) ; 7.15(1 \mathrm{H}$, ddd, 1.6, 7.6, 7.9, 12-H); $6.89(2 \mathrm{H}, \mathrm{m}, 13-\mathrm{H}, 11-\mathrm{H}) ; 6.00\left(2 \mathrm{H}, \mathrm{s}, \mathrm{CH}_{2}\right) ; 3.84(3 \mathrm{H}, \mathrm{s}, \mathrm{OMe}) .{ }^{13} \mathrm{C}-$ NMR $\delta 163.38$ (CO); 150.73 (1-C); 150.13 (10-C); 138.23 (6-C); 137.95 (4a-C); 135.30 (3-C); 132.00 (7-C); 130.75 (8-C); 127.56 (8a-C); 127.30 (5-C); 126.97 (12-C); 126.18 (4-C); 124.78 (9-C); 122.14 (14-C); 120.79 (13-C); 111.00 (11-C); $62.84\left(\mathrm{CH}_{2}\right) ; 55.65(\mathrm{OMe})$. Anal. calcd. for $\mathrm{C}_{18} \mathrm{H}_{17} \mathrm{ClN}_{2} \mathrm{O}_{2}$ (328.79): C, 65.75; H, 5.21; N, 8.52\%. Found: C, 65.78; H, 5.26; N, 8.48\%.

2-[N-(3-Trifluorophenyl)carbamoylmethyl]isoquinolinium chloride (3d). IR $v$ 3198, 3056, 1693, 1577. ${ }^{1} \mathrm{H}-\mathrm{NMR}(\delta \mathrm{ppm}, J \mathrm{~Hz}): 10.16(1 \mathrm{H}, \mathrm{s}, \mathrm{NH}), 9.69(1 \mathrm{H}, \mathrm{bs}, \mathrm{H}-1) ; 8.52(1 \mathrm{H}, \mathrm{dd}, 1.3$, 6.9, 3-H); 8.42 (1H, bd, 8.4, 8-H); $8.33(1 \mathrm{H} \mathrm{d}, 6.9,4-\mathrm{H}) ; 8.28(1 \mathrm{H}$, ddd, 1.2, 6.7, 7.3, 6-H); 8.21 $(1 \mathrm{H}, \mathrm{bd}, 7.3,5-\mathrm{H}) ; 8.08(1 \mathrm{H}, \mathrm{ddd}, 1.4,6.7,8.4,7-\mathrm{H}) ; 7.91(1 \mathrm{H}, \mathrm{bs}, 10-\mathrm{H}) ; 7.66(1 \mathrm{H}, \mathrm{m}, 12-\mathrm{H})$; $7.47(1 \mathrm{H}, \mathrm{t}, 7.7,13-\mathrm{H}) ; 7.45(1 \mathrm{H}, \mathrm{m}, 14-\mathrm{H}) ; 5.97\left(2 \mathrm{H}, \mathrm{s}, \mathrm{CH}_{2}\right) .{ }^{13} \mathrm{C}-\mathrm{NMR} \delta 163.39(\mathrm{CO}) ; 150.61$ (1-C); 138.45 (6-C); 138.00 (4a-C); 136.52 (9-C); 135.28 (3-C); 132.21 (7-C); 131.68 (11-C, q, 32.8); 130.80 (8-C); 129.86 (C13-H); 127.62 (8a-C); 127.35 (5-C); 126.24 (4-C); 123.91 (14-C); $123.69\left(\mathrm{CF}_{3}, \mathrm{q}, 271.8\right) ; 122.77$ (12-C, q, 3.4); 117.65 (10-C, q, 3.7); $62.73\left(\mathrm{CH}_{2}\right)$. Anal. calcd. for $\mathrm{C}_{18} \mathrm{H}_{14} \mathrm{ClF}_{3} \mathrm{~N}_{2} \mathrm{O}$ (366.76): C, 58.95; H, 3.85; N, 7.64\%. Found: C, 59.02; H, 3.75; N, 7.61\%.

\section{Carbamoyl-substituted indolizines and benzoindolizines. General procedure}

A mixture of N-methylcarbamoyl quaternary salt $(10 \mathrm{mmol})$ and acetylenic compound (15 mmol) in propenoxid $(50 \mathrm{~mL})$ was stirred at room temperature for 10-12 days and then was concentrated under reduced pressure. The residue was treated with methanol $(10 \mathrm{~mL})$ and kept refrigerated overnight. The solid was filtered and washed with cold methanol and then with diethyl ether. All crude products were recrystallised from chloroform/methanol. 
The yields and m. p. for 3-carbamoylindolizines 5-12 are shown in Table 2. The spectral data are given below.

1-Carbethoxy-3-[(2-fluorophenyl)carbamoyl]indolizine (5). IR $v$ 3323, 3112, 1669, 1656, 1529. ${ }^{1} \mathrm{H}-\mathrm{NMR}(\delta \mathrm{ppm}, J \mathrm{~Hz}): 9.71(1 \mathrm{H}, \mathrm{dt}, 7.1,1.1,5-\mathrm{H}) ; 8.40(1 \mathrm{H}, \mathrm{m}, 14-\mathrm{H}) ; 8.34(1 \mathrm{H}, \mathrm{dt}, 9.1$, 1.3, 8-H); $7.93(1 \mathrm{H}, \mathrm{d}, 3.1, \mathrm{NH}) ; 7.85(1 \mathrm{H}, \mathrm{s}, 2-\mathrm{H}) ; 7.33(1 \mathrm{H}, \mathrm{ddd}, 1.1,6.8,9.1,7-\mathrm{H})$; 7.04-7.22 $(3 \mathrm{H}, \mathrm{m}, 12-\mathrm{H}, 13-\mathrm{H}, 15-\mathrm{H}) ; 4.40\left(2 \mathrm{H}, \mathrm{q}, 7.1, \mathrm{CH}_{2}\right.$ from $\left.\mathrm{CO}_{2} \mathrm{Et}\right) ; 1.43\left(3 \mathrm{H}, \mathrm{t}, 7.1, \mathrm{CH}_{3}\right.$ from $\left.\mathrm{CO}_{2} \mathrm{Et}\right) .{ }^{13} \mathrm{C}-\mathrm{NMR} \delta 164.07$ (COO); 159.14 (9-C); 152.70 (11-C, d, 243.1); 138.73 (8a-C); 128.25 (5-C); 125.95 (10-C, d, 10.3); 124.55 (15-C or 13-C, d, 3.4); 124.22 (13-C or 15-C, d, 7.5); 121.78 (14-C); 119.47 (8-C); 119.47 (2-C); 116.80 (3-C); 114.92 (12-C, d, 19.1); 114.32 (6-C); 104.85 (1-C); $60.06\left(\mathrm{CH}_{2}\right.$ from $\left.\mathrm{CO}_{2} \mathrm{Et}\right) ; 14.54\left(\mathrm{CH}_{3}\right.$ from $\left.\mathrm{CO}_{2} \mathrm{Et}\right)$. Anal. calcd. for $\mathrm{C}_{19} \mathrm{H}_{16} \mathrm{BrF}_{3} \mathrm{~N}_{2} \mathrm{O}$ (425.24): C, 53.66; H, 3.79; N, 6.59\%. Found: C, 53.60; H, 3.85; N, 6.61\%. Anal. calcd. for $\mathrm{C}_{18} \mathrm{H}_{15} \mathrm{FN}_{2} \mathrm{O}_{3}$ (326.32): C, 66.25; H, 4.63; N, 8.58\%. Found: C, 66.30; H, 4.65; $\mathrm{N}, 8.63 \%$.

1-Carbethoxy-3-[(2-ethylphenyl)carbamoyl]indolizine (6). IR $v$ 3336, 3110, 1671, 1653, 1528. ${ }^{1} \mathrm{H}-\mathrm{NMR}(\delta \mathrm{ppm}, J \mathrm{~Hz}): 9.72(1 \mathrm{H}, \mathrm{dt}, 7.2,1.1,5-\mathrm{H}) ; 8.29(1 \mathrm{H}, \mathrm{dt}, 1.3,8.9,8-\mathrm{H}) ; 7.86(1 \mathrm{H}$, s, 2-H); $7.80(1 \mathrm{H}, \mathrm{dd}, 7.8,1.8,15-\mathrm{H}) ; 7.72(1 \mathrm{H}, \mathrm{s}, \mathrm{NH}) ; 7.23-7.32(3 \mathrm{H}, \mathrm{m}, 7-\mathrm{H}, 12-\mathrm{H}, 14-\mathrm{H})$; $7.20(1 \mathrm{H}, \mathrm{dt}, 7.0,1.5,13-\mathrm{H}) ; 6.94(1 \mathrm{H}, \mathrm{td}, 6.9,1.4,6-\mathrm{H}) ; 4.37\left(2 \mathrm{H}, \mathrm{CH}_{2}, \mathrm{q}, 7.1\right.$, from $\left.\mathrm{CO}_{2} \mathrm{Et}\right)$; $2.70\left(2 \mathrm{H}, \mathrm{q}, 7.4, \mathrm{CH}_{2}\right.$ from 2-Et); $1.41\left(3 \mathrm{H}, \mathrm{t}, 7.1, \mathrm{CH}_{3}\right.$ from $\left.\mathrm{CO}_{2} \mathrm{Et}\right) ; 1.28\left(3 \mathrm{H}, \mathrm{t}, 7.4, \mathrm{CH}_{3}\right.$ from 2-Et). ${ }^{13} \mathrm{C}-\mathrm{NMR} \delta 164.32$ (COO); 159.84 (9-C); 138.51 (8a-C); 136.09 (10-C); 134.69 (11-C); 128.65 (12-C); 128.46 (5-C); 126.67 (7-C); 125.82 (13-C); 125.43 (14-C); 124.42 (15-C); 119.45 (8-C); 119.02 (2-C); 117.36 (3-C), 114.14 (6-C); 104.65 (1-C); $60.06\left(\mathrm{CH}_{2}\right.$ from $\left.\mathrm{CO}_{2} \mathrm{Et}\right) ; 24.43$ $\left(\mathrm{CH}_{2}\right.$ from 2-Et); $14.55\left(\mathrm{CH}_{3}\right.$ from $\left.\mathrm{CO}_{2} \mathrm{Et}\right) ; 13.98\left(\mathrm{CH}_{3}\right.$ from 2-Et). Anal. calcd. for $\mathrm{C}_{20} \mathrm{H}_{20} \mathrm{~N}_{2} \mathrm{O}_{3}$ (336.38): C, 71.41; H, 5.99; N, 8.33\%. Found: C, 71.38; H, 6.04; N, 8.28\%.

1-Carbethoxy-3-[(3-trifluoromethylphenyl)carbamoyl]indolizine (7). IR $\vee$ 3326, 3123, 1669, 1657, 1554. ${ }^{1} \mathrm{H}-\mathrm{NMR}(\delta \mathrm{ppm}, J \mathrm{~Hz}): 9.69(1 \mathrm{H}, \mathrm{dt}, 7.2,1.1,5-\mathrm{H}) ; 8.29(1 \mathrm{H}, \mathrm{dt}, 9.1,1.1,8-\mathrm{H})$; $8.16(1 \mathrm{H}, \mathrm{s}, \mathrm{NH}) ; 8.01(1 \mathrm{H}, \mathrm{bs}, 11-\mathrm{H}) ; 7.93(1 \mathrm{H}, \mathrm{s}, 2-\mathrm{H}) ; 7.83(1 \mathrm{H}, \mathrm{bd}, 7.8,13-\mathrm{H}) ; 7.42(1 \mathrm{H}, \mathrm{t}$, 7.8, 14-H); $7.37(1 \mathrm{H}, \mathrm{bd}, 7.8,15-\mathrm{H}) ; 7.31(1 \mathrm{H}, \mathrm{dd}, 9.1,7.2,7-\mathrm{H}) ; 6.97(1 \mathrm{H}, \mathrm{td}, 7.2,0.9,6-\mathrm{H})$; $4.37\left(2 \mathrm{H}, \mathrm{q}, 7.2, \mathrm{CH}_{2}\right.$ from $\left.\mathrm{CO}_{2} \mathrm{Et}\right) ; 1.42\left(3 \mathrm{H}, \mathrm{t}, 7.2, \mathrm{CH}_{3}\right.$ from $\left.\mathrm{CO}_{2} \mathrm{Et}\right) .{ }^{13} \mathrm{C}-\mathrm{NMR} \delta 164.28$ (COO); 159.51 (9-C); 138.74 (8a-C); 138.52 (10-C); 131.58 (12-C, q, 31.9); 129.57 (14-C); 128.34 (5-C); 125.84 (7-C); $123.81\left(\mathrm{CF}_{3}, \mathrm{q}, 272.3\right) ; 123.07$ (15-C); 120.69 (13-C, q, 3.4); 119.77 (2-C); 119.51 (8-C); 116.79 (11-C, q, 3.7); 116.42 (3-C); 114.43 (6-C); 104.83 (1-C); $60.22\left(\mathrm{CH}_{2}\right.$ from $\left.\mathrm{CO}_{2} \mathrm{Et}\right) ; 14.56\left(\mathrm{CH}_{3}\right.$ from $\left.\mathrm{CO}_{2} \mathrm{Et}\right)$. Anal. calcd. for $\mathrm{C}_{19} \mathrm{H}_{15} \mathrm{~F}_{3} \mathrm{~N}_{2} \mathrm{O}_{3}$ (376.33): C, 60.64; $\mathrm{H}$, 4.02; N, 7.44\%. Found: C, 60.58; H, 4.05; N, 7.38\%.

1-Carbethoxy-3-[(2-fluorophenyl)carbamoyl]-7-methylindolizine (8). IR v 3319, 3122, 1691, 1631, 1534. ${ }^{1} \mathrm{H}-\mathrm{NMR}(\delta \mathrm{ppm}, J \mathrm{~Hz}): 9.59(1 \mathrm{H}, \mathrm{dd}, 7.2,1.0,5-\mathrm{H}) ; 8.37(1 \mathrm{H}, \mathrm{td}, 8.1,1.7,14-\mathrm{H})$; $8.11(1 \mathrm{H}$, dqui, 2.0, 1.0, 8-H); $7.87(1 \mathrm{H}, \mathrm{d}, 3.2, \mathrm{NH}) ; 7.78(1 \mathrm{H}, \mathrm{s}, 2-\mathrm{H}) ; 7.04-7.20(3 \mathrm{H}, \mathrm{m}, 12-\mathrm{H}$, 13-H, 15-H); $4.39\left(2 \mathrm{H}, \mathrm{q}, 7.1, \mathrm{CH}_{2}\right.$ from $\left.\mathrm{CO}_{2} \mathrm{Et}\right) ; 2.45\left(3 \mathrm{H}, \mathrm{s}, 7-\mathrm{CH}_{3}\right) ; 1.44\left(3 \mathrm{H}, \mathrm{t}, 7.1, \mathrm{CH}_{3}\right.$ from $\left.\mathrm{CO}_{2} \mathrm{Et}\right) .{ }^{13} \mathrm{C}-\mathrm{NMR} \delta 164.22$ (COO); 159.19 (9-C); 152.63 (11-C, d, 241.6); 139.32 (8a-C); 137.17 (7-C); 127.65 (5-C); 126.45 (10-C, d, 10.0); 124.56 (13-C or 15-C, d, 3.6); 124.10 (15-C or 13-C, d, 7.8); 121.65 (14-C); 119.53 (2-C); 118.11 (8-C); 116.93 (6-C); 116.25 (3-C); 114.91 (12-C, d, 19.5); 103.53 (1-C); $59.96\left(\mathrm{CH}_{2}\right.$ from $\left.\mathrm{CO}_{2} \mathrm{Et}\right) ; 21.42\left(7-\mathrm{CH}_{3}\right) ; 14.52\left(\mathrm{CH}_{3}\right.$ from $\left.\mathrm{CO}_{2} \mathrm{Et}\right)$. 
Anal. calcd. for $\mathrm{C}_{19} \mathrm{H}_{17} \mathrm{FN}_{2} \mathrm{O}_{3}$ (340.35): C, 67.05; H, 5.03; N, 8.23\%. Found: $\mathrm{C}, 67.10 ; \mathrm{H}, 5.15$; $\mathrm{N}, 8.26 \%$.

1-Carbethoxy-3-[(3-trifluoromethylphenyl)carbamoyl]indolizine (9). IR $\vee$ 3361, 3117, 1673, 1657, 1553. ${ }^{1} \mathrm{H}-\mathrm{NMR}(\delta \mathrm{ppm}, J \mathrm{~Hz}): 9.58(1 \mathrm{H}, \mathrm{dt}, 7.2,0.8,5-\mathrm{H}) ; 8.10(1 \mathrm{H}, \mathrm{dq}, 2.0,1.0,8-\mathrm{H})$; $8.03(1 \mathrm{H}, \mathrm{s}, \mathrm{NH}) ; 7.98(1 \mathrm{H}, \mathrm{bs}, 11-\mathrm{H}) ; 7.83(1 \mathrm{H}, \mathrm{s}, 2-\mathrm{H}) ; 7.78(1 \mathrm{H}, \mathrm{dq}, 7.8,1.5,13-\mathrm{H}) ; 7.47(1 \mathrm{H}$, t, 7.8, 14-H); $7.39(1 \mathrm{H}, \mathrm{bd}, 7.8,15-\mathrm{H}) ; 6.82(1 \mathrm{H}, \mathrm{dd}, 7.2,2.0,6-\mathrm{H}) ; 4.37\left(2 \mathrm{H}, \mathrm{q}, 7.1, \mathrm{CH}_{2}\right.$ from $\left.\mathrm{CO}_{2} \mathrm{Et}\right) ; 2.43\left(3 \mathrm{H}, \mathrm{d}, 1.0,7-\mathrm{CH}_{3}\right) ; 1.43\left(3 \mathrm{H}, \mathrm{t}, 7.1, \mathrm{CH}_{3}\right.$ from $\left.\mathrm{CO}_{2} \mathrm{Et}\right) .{ }^{13} \mathrm{C}-\mathrm{NMR} \delta 164.37(\mathrm{COO})$; 159.53 (9-C); 139.33 (8a-C); 138.61 (10-C); 137.32 (7-C); 131.50 (12-C, q, 32.1); 129.55 (14C); 127.70 (5-C); $123.81\left(\mathrm{CF}_{3}, \mathrm{q}, 271.8\right) ; 122.99$ (15-C); 120.56 (13-C, q, 3.4); 119.78 (2-C); 118.13 (8-C); 116.98 (6-C); 116.74 (11-C, q, 3.8); 116.18 (3-C); 103.53 (1-C); 60.05 ( $\mathrm{CH}_{2}$ from $\left.\mathrm{CO}_{2} \mathrm{Et}\right) ; 21.44\left(7-\mathrm{CH}_{3}\right) ; 14.56\left(\mathrm{CH}_{3}\right.$ from $\left.\mathrm{CO}_{2} \mathrm{Et}\right)$. Anal. calcd. for $\mathrm{C}_{20} \mathrm{H}_{17} \mathrm{~F}_{3} \mathrm{~N}_{2} \mathrm{O}_{3}$ (390.36): C, 61.54; H, 4.39; N, 7.18\%. Found: C, 61.48; H, 4.45; N, 7.12\%.

1-Benzoyl-3-[(2-methyl-6-ethylphenyl)carbamoyl]-7-methylindolizine (10). IR v 3295, 3134, 1656, 1637, 1511. ${ }^{1} \mathrm{H}-\mathrm{NMR}(\delta \mathrm{ppm}, J \mathrm{~Hz}): 9.63(1 \mathrm{H}, \mathrm{d}, 7.2,5-\mathrm{H}) ; 8.36(1 \mathrm{H}$, dqui, 1.8, 0.9, 8-H8); 7.97 (1H, bs, NH); $7.80(2 \mathrm{H}, \mathrm{m}, \mathrm{H}-$ orto from $\mathrm{Ph}) ; 7.79(1 \mathrm{H}, \mathrm{s}, 2-\mathrm{H}) ; 7.45$ (3H, m, H-meta, Hpara from $\mathrm{Ph}) ; 7.08-7.20(3 \mathrm{H}, \mathrm{m}, 12-\mathrm{H}, 13-\mathrm{H}, 14-\mathrm{H}) ; 6.83(1 \mathrm{H}, \mathrm{dd}, 7.2,1.8,6-\mathrm{H}) ; 2.61(2 \mathrm{H}, \mathrm{q}$, 7.6, $\mathrm{CH}_{2}$ from Et); $2.44\left(3 \mathrm{H}, \mathrm{s}, 7-\mathrm{CH}_{3}\right) ; 2.24\left(3 \mathrm{H}, \mathrm{s}, 2-\mathrm{CH}_{3}\right) ; 1.15\left(3 \mathrm{H}, \mathrm{t}, 7.6, \mathrm{CH}_{3}\right.$ from Et). ${ }^{13} \mathrm{C}-$ NMR $\delta 190.20$ (1-COPh); 160.32 (9-C); 141.51 (7-C); 140.59 (8a-C); 140.01 (10-C); 138.56 (15-C); 136.22 (11-C); $132.83\left(\mathrm{C}_{\mathrm{q}}-\mathrm{Ph}\right) ; 130.94$ (C-para from $\left.\mathrm{Ph}\right) ; 128.65$ (2C-ortho from $\left.\mathrm{Ph}\right)$; 128.18 (2C-meta from $\mathrm{Ph}$ ); 127.91 (14-C or 12-C); 127.68 (12-C or 14-C); 126.32 (13-C); 121.06 (2-C); 118.99 (8-C); 117.71 (6-C); 116.73 (3-C); 111.18 (1-C); $24.86\left(\mathrm{CH}_{2}\right.$ from Et); 21.39 (2-Me); 18.46 (7-Me); 14.36 ( $\mathrm{CH}_{3}$ from Et). Anal. calcd. for $\mathrm{C}_{26} \mathrm{H}_{24} \mathrm{~N}_{2} \mathrm{O}_{2}$ (396.49): C, 78.76; H, 6.10; N, 7.06\%. Found: C, 78.83; H, 6.18; N, 7.98\%.

1-Acetyl-3-[(2,6-diethylphenyl)carbamoyl]-5-methyl-8-ethylindolizine (11). IR v 3168, 1663, 1631, 1512. ${ }^{1} \mathrm{H}-\mathrm{NMR}(\delta \mathrm{ppm}, J \mathrm{~Hz}): 7.85(1 \mathrm{H}, \mathrm{s}, 2-\mathrm{H}) ; 7.65(1 \mathrm{H}, \mathrm{s}, \mathrm{NH}) ; 7.27(1 \mathrm{H}, \mathrm{m}, 13-\mathrm{H})$; $7.16(2 \mathrm{H}, \mathrm{m}, 12-\mathrm{H}, 14-\mathrm{H}) ; 7.02(1 \mathrm{H}, \mathrm{d}, 7.2,7-\mathrm{H}) ; 6.66(1 \mathrm{H}, \mathrm{d}, 7.2,6-\mathrm{H}) ; 3.20\left(2 \mathrm{H}, \mathrm{q}, 7.4, \mathrm{CH}_{2}\right.$ from 8-Et); 2.70 (4H, q, 7.7, 2 $\mathrm{CH}_{2}$ from 2,6-diEt); $2.62(3 \mathrm{H}, \mathrm{s}, 5-\mathrm{Me}) ; 2.57\left(3 \mathrm{H}, \mathrm{s}, \mathrm{CH}_{3}\right.$ from 1 $\mathrm{Ac}) ; 1.27\left(6 \mathrm{H}, \mathrm{t}, 7.7,2 \mathrm{CH}_{3}\right.$ from 2,6-diEt); $1.10\left(3 \mathrm{H}, \mathrm{t}, 7.4, \mathrm{CH}_{3}\right.$ from 8 -Et). ${ }^{13} \mathrm{C}-\mathrm{NMR} \delta 192.58$ (CO-Ac); 161.12 (9-C); 141.67 (5-C); 137.54 (8a-C); 135.30 (10-C); 134.18 (11-C, 15-C); 132.13 (8-C); 128.23 (7-C); 126.56 (12-C, 14-C); 125.73 (13-C); 123.59 (2-C); 120.24 (3-C); 116.22 (6-C); 103.30 (1-C); $29.45\left(\mathrm{CH}_{3}\right.$ from Ac); $27.25\left(\mathrm{CH}_{2}\right.$ from 8-Et); $24.91\left(2 \mathrm{CH}_{2}\right.$ from 2,6-diEt); 21.91 (5-Me); $14.61\left(3 \mathrm{CH}_{3}\right.$ from 8-Et, 2-Et, 6-Et). Anal. calcd. for $\mathrm{C}_{24} \mathrm{H}_{28} \mathrm{~N}_{2} \mathrm{O}_{2}$ (376.50): C, 76.56; H, 7.50; N, 7.44\%. Found: C, 76.61; H, 7.56; N, 7.46\%.

1-Carbomethoxy-3-[(2,6-diethylphenyl)carbamoyl]-5-methyl-8-ethylindolizine (12). IR $\mathrm{v}$ 3359, 3273, 1698, 1648, 1504. ${ }^{1} \mathrm{H}-\mathrm{NMR}\left(\mathrm{CDCl}_{3}, \delta \mathrm{ppm}, J \mathrm{~Hz}\right): 7.83(1 \mathrm{H}, \mathrm{s}, 2-\mathrm{H}) ; 7.47(1 \mathrm{H}, \mathrm{s}$, $\mathrm{NH}) ; 7.27(1 \mathrm{H}, \mathrm{m}, 13-\mathrm{H}) ; 7.16(2 \mathrm{H}, \mathrm{m}, 12-\mathrm{H}, 14-\mathrm{H}) ; 7.03(1 \mathrm{H}, \mathrm{d}, 7.3,7-\mathrm{H}) ; 6.66(1 \mathrm{H}, \mathrm{d}, 7.3,6-$ $\mathrm{H}) ; 3.90$ (3H, s, Me from $\left.\mathrm{CO}_{2} \mathrm{Me}\right) ; 3.24\left(2 \mathrm{H}, \mathrm{q}, 7.4, \mathrm{CH}_{2}\right.$ from 8-Et); 2.69 (4H, q, 7.6, 2CH $\mathrm{CH}_{2}$ from 2,6-diEt); $2.64(3 \mathrm{H}, \mathrm{s}, 5-\mathrm{Me}) ; 1.26\left(6 \mathrm{H}, \mathrm{t}, 7.6,2 \mathrm{CH}_{3}\right.$ from 2,6-diEt); $1.22\left(3 \mathrm{H}, \mathrm{t}, 7.4, \mathrm{CH}_{3}\right.$ from 8-Et). ${ }^{13} \mathrm{C}-\mathrm{NMR} \delta 164.61$ (COO); 160.98 (9-C); 141.76 (5-C); 138.47 (8a-C); 135.63 (10-C); 133.48 (11-C, 15-C); 132.05 (8-C); 128.26 (7-C); 126.51 (12-C, 14-C); 125.12 (13-C); 123.78 (2-C); 120.19 (3-C); 115.74 (6-C); 105.21 (1-C); $51.54\left(\mathrm{CH}_{3}\right.$ from $\left.\mathrm{CO}_{2} \mathrm{Me}\right) 27.00\left(\mathrm{CH}_{2}\right.$ from 8Et); $24.87\left(\mathrm{CH}_{2}\right.$ from 2,6-diEt); 22.09 (5-Me); $14.96\left(\mathrm{CH}_{3}\right.$ from 8-Et); $14.57\left(2 \mathrm{CH}_{3}\right.$ from 2,6- 
diEt). Anal. calcd. for $\mathrm{C}_{24} \mathrm{H}_{28} \mathrm{~N}_{2} \mathrm{O}_{3}$ (392.50): C, 73.44; H, 7.19; N, 7.14\%. Found: C, 73.49; H, $7.16 ; \mathrm{N}, 7.08 \%$.

\section{1-[(3-Trifluoromethylphenyl)carbamoyl]-3-carbethoxy-7-methylpyrrolo[1,2-a]quinoline} (13). m. p.: $182-184^{\circ} \mathrm{C}$; yield: $23 \%$; IR $\vee 3254,1702,1649,1544 .{ }^{1} \mathrm{H}-\mathrm{NMR}(\delta \mathrm{ppm}, J \mathrm{~Hz}): 8.02$ $(1 \mathrm{H}, \mathrm{bs}, 13-\mathrm{H}) ; 7.96(1 \mathrm{H}, \mathrm{bd}, 8.0,17-\mathrm{H}) ; 7.93(1 \mathrm{H}, \mathrm{d}, 9.4,5-\mathrm{H}) ; 7.86(1 \mathrm{H}, \mathrm{d}, 9.3,9-\mathrm{H}) ; 7.56(1 \mathrm{H}$, s, 2-H); $7.53(1 \mathrm{H}, \mathrm{t}, 8.0,16-\mathrm{H}) ; 7.47(1 \mathrm{H}, \mathrm{bd}, 8.0,15-\mathrm{H}) ; 7.37(1 \mathrm{H}, \mathrm{s}, \mathrm{NH}) ; 7.30-7.40(2 \mathrm{H}, \mathrm{m}, 6-$ $\mathrm{H}, 8-\mathrm{H}) ; 7.20(1 \mathrm{H}, \mathrm{d}, 9.4,4-\mathrm{H}) ; 4.39\left(2 \mathrm{H}, \mathrm{q}, 7.1, \mathrm{CH}_{2}\right.$ from $\left.\mathrm{CO}_{2} \mathrm{Et}\right) ; 2.44\left(3 \mathrm{H}, \mathrm{s}, 7-\mathrm{CH}_{3}\right) ; 1.43$ $\left(3 \mathrm{H}, \mathrm{t}, 7.1, \mathrm{CH}_{3}\right.$ from $\left.\mathrm{CO}_{2} \mathrm{Et}\right) .{ }^{13} \mathrm{C}-\mathrm{NMR} \delta 165.75(\mathrm{COO}) ; 162.18$ (11-C); 137.93 (3a-C, 9a-C); 137.84 (12-C); 135.27 (7-C); 131.54 (14-C, q, 33.1); 130.24 (5-C); 130.07 (8-C); 129.72 (16-C); 128.35 (6-C); 127.59 (4-C); 124.39 (1-C); 123.75 (15-C, q, 3.4); $123.56\left(\mathrm{CF}_{3}, \mathrm{q}, 279.1\right) ; 121.86$ (2-C); 121.85 (17-C); 118.26 (9-C); 117.43 (13-C, q, 3.5); 116.50 (5-C); 105.67 (3-C); 61.13 $\left(\mathrm{CH}_{2}\right.$ from $\left.\mathrm{CO}_{2} \mathrm{Et}\right) ; 20.69\left(7-\mathrm{CH}_{3}\right) ; 14.11\left(\mathrm{CH}_{3}\right.$ from $\left.\mathrm{CO}_{2} \mathrm{Et}\right)$. Anal. calcd. for $\mathrm{C}_{24} \mathrm{H}_{19} \mathrm{~F}_{3} \mathrm{~N}_{2} \mathrm{O}_{3}$ (440.42): C, 65.45; H, 4.35; N, 6.36\%. Found: C, 65.51; H, 4.42; N, 6.44\%.

The yields and m. p. for 3-carbamoylpyrrolo[2,1-a]isoquinolines 14-29 are shown in Table 3. The spectral data are given below.

1-Carbethoxy-3-[(2-fluorophenyl)carbamoyl]pyrrolo[2,1-a]isoquinoline (14). IR $v$ 3306, 3055, 1700, 1669, 1534. ${ }^{1} \mathrm{H}-\mathrm{NMR}(\delta \mathrm{ppm}, J \mathrm{~Hz}): 9.77(1 \mathrm{H}, \mathrm{dd}, 7.3,2.2,10-\mathrm{H}) ; 9.37(1 \mathrm{H}, \mathrm{d}, 7.5$, 5-H); $8.36(1 \mathrm{H}, \mathrm{td}, 8.0,1.6,17-\mathrm{H}) ; 7.99(1 \mathrm{H}, \mathrm{s}, \mathrm{NH}) ; 7.80(1 \mathrm{H}, \mathrm{s}, 2-\mathrm{H}) ; 7.67(1 \mathrm{H}, \mathrm{dd}, 7.0,2.4,7-$ $\mathrm{H})$; 7.53-7.64 (2H, m, 8-H, H-9); 7.05-7.20 (3H, m, 14-H, 15-H, 16-H); $7.11(1 \mathrm{H}, \mathrm{d}, 7.5,6-\mathrm{H})$; $4.43\left(2 \mathrm{H}, \mathrm{q}, 7.1, \mathrm{CH}_{2}\right.$ from $\left.\mathrm{CO}_{2} \mathrm{Et}\right) ; 1.46\left(3 \mathrm{H}, \mathrm{t}, 7.1, \mathrm{CH}_{3}\right.$ from $\left.\mathrm{CO}_{2} \mathrm{Et}\right) .{ }^{13} \mathrm{C}-\mathrm{NMR} \delta 164.42$ (COO); 159.02 (11-C); 152.70 (13-C, d, 243.3); 135.66 (10b-C); 129.55 (6a-C); 128.78 (8-C or 9-C); 127.67 (10-C); 127.59 (9-C or 8-C); 126.60 (7-C); 126.27 (12-C, d, 10.2); 124.76 (10a-C); 124.60 (16-C, d, 3.6); 124.43 (5-C); 124.38 (15-C, d, 7.5); 121.83 (17-C); 120.20 (2-C); 118.25 (3-C); 114.94 (14-C, d, 18.3); 114.81 (6-C); 109.11 (1-C); $60.60\left(\mathrm{CH}_{2}\right.$ from $\left.\mathrm{CO}_{2} \mathrm{Et}\right) ; 14.49\left(\mathrm{CH}_{3}\right.$ from $\mathrm{CO}_{2} \mathrm{Et}$ ). Anal. calcd. for $\mathrm{C}_{22} \mathrm{H}_{17} \mathrm{FN}_{2} \mathrm{O}_{3}$ (376.39): C, 70.20; H, 4.55; N, 7.44\%. Found: $\mathrm{C}$, $70.26 ; \mathrm{H}, 4.66 ; \mathrm{N}, 7.54 \%$.

1-Carbomethoxy-3-[(3-methylphenyl)carbamoyl]pyrrolo[2,1-a]isoquinoline (15). IR $\vee 3311$, 3134, 1710, 1632, 1533. ${ }^{1} \mathrm{H}-\mathrm{NMR}(\delta \mathrm{ppm}, J \mathrm{~Hz}): 9.73(1 \mathrm{H}, \mathrm{m}, 10-\mathrm{H}) ; 9.29(1 \mathrm{H}, \mathrm{d}, 7.5,5-\mathrm{H})$; $7.91(1 \mathrm{H}, \mathrm{s}, \mathrm{NH}) ; 7.73(1 \mathrm{H}, \mathrm{s}, 2-\mathrm{H}) ; 7.64(1 \mathrm{H}, \mathrm{m}, 7-\mathrm{H}) ; 7.52-7.59(2 \mathrm{H}, \mathrm{m}, 8-\mathrm{H}, 9-\mathrm{H}) ; 7.50(1 \mathrm{H}$, bs, 13-H); $7.40(1 \mathrm{H}, \mathrm{bd}, 7.6,17-\mathrm{H}) ; 7.25(1 \mathrm{H}, \mathrm{t}, 7.6,16-\mathrm{H}) ; 7.04(1 \mathrm{H}, \mathrm{d}, 7.5,6-\mathrm{H}) ; 6.97(1 \mathrm{H}, \mathrm{bd}$, 7.6, $15-\mathrm{H}) ; 3.92\left(3 \mathrm{H}, \mathrm{s}, \mathrm{CH}_{3}\right.$ from $\left.\mathrm{CO}_{2} \mathrm{Me}\right) ; 2.37\left(3 \mathrm{H}, \mathrm{s}, 3-\mathrm{CH}_{3}\right) .{ }^{13} \mathrm{C}-\mathrm{NMR} \delta 164.94(\mathrm{COO})$; 159.30 (11-C); 139.04 (12-C); 137.70 (14-C); 135.51 (10b-C); 129.55 (6a-C); 128.91 (16-C); 128.74 (8-C or 9-C); 127.56 (10-C); 127.55 (9-C or 8-C); 126.61 (7-C); 125.26 (15-C); 124.77 (10a-C); 124.45 (5-C); 120.86 (17-C); 119.74 (2-C); 118.98 (3-C); 117.29 (13-C); 114.67 (6-C); 108.37 (1-C); $51.65\left(\mathrm{CH}_{3}\right.$ from $\left.\mathrm{CO}_{2} \mathrm{Me}\right) ; 21.51$ (3-Me). Anal. calcd. for $\mathrm{C}_{22} \mathrm{H}_{18} \mathrm{~N}_{2} \mathrm{O}_{3}$ (358.40): C, $73.73 ; \mathrm{H}, 5.06 ; \mathrm{N}, 7.82 \%$. Found: C, 73.80; H, 5.98; N, 7.86\%.

1-Carbethoxy-3-[(2-methoxyphenyl)carbamoyl]pyrrolo[2,1-a]isoquinoline (16). IR $\vee 3331$, 3126, 1703, 1669, 1532. ${ }^{1} \mathrm{H}-\mathrm{NMR}(\delta \mathrm{ppm}, J \mathrm{~Hz}): 9.80(1 \mathrm{H}, \mathrm{dd}, 7.5,2.0,10-\mathrm{H}) ; 9.50(1 \mathrm{H}, \mathrm{d}, 7.6$, 5-H); $8.45(1 \mathrm{H}, \mathrm{s}, \mathrm{NH}) ; 8.42(1 \mathrm{H}, \mathrm{dd}, 7.9,1.7,17-\mathrm{H}) ; 7.83(1 \mathrm{H}, \mathrm{s}, 2-\mathrm{H}) ; 7.73(1 \mathrm{H}, \mathrm{dd}, 7.3,2.2,7-$ $\mathrm{H}) ; 7.58-7.65(2 \mathrm{H}, \mathrm{m}, 8-\mathrm{H}, 9-\mathrm{H}) ; 7.17(1 \mathrm{H}, \mathrm{d}, 7.6,6-\mathrm{H}) ; 7.11(1 \mathrm{H}, \mathrm{td}, 7.9,1.7,15-\mathrm{H}) ; 7.03(1 \mathrm{H}$, td, 7.9, 1.6, 16-H); $6.96(1 \mathrm{H}, \mathrm{dd}, 7.9,1.6,14-\mathrm{H}) ; 4.45\left(2 \mathrm{H}, \mathrm{q}, 7.1, \mathrm{CH}_{2}\right.$ from $\left.\mathrm{CO}_{2} \mathrm{Et}\right) ; 3.98(3 \mathrm{H}, \mathrm{s}$, $\mathrm{OMe}) ; 1.49\left(3 \mathrm{H}, \mathrm{t}, 7.1, \mathrm{CH}_{3}\right.$ from $\left.\mathrm{CO}_{2} \mathrm{Et}\right) .{ }^{13} \mathrm{C}-\mathrm{NMR} \delta 164.65(\mathrm{COO}) ; 159.09$ (11-C); 148.14 
(13-C); 135.38 (10b-C); 129.59 (6a-C, 12-C); 128.64 (9-C or 8-C); 127.62 (10-C); 127.51 (8-C or 9-C); 126.63 (7-C); 124.93 (10a-C); 124.69 (5-C); 123.81 (15-C); 121.14 (16-C); 119.93 (17C); 119.74 (2-C); 119.24 (3-C); 114.62 (6-C); 110.06 (14-C); 108.97 (1-C); $60.53\left(\mathrm{CH}_{2}\right.$ from $\left.\mathrm{CO}_{2} \mathrm{Et}\right) ; 55.56(\mathrm{OMe}) ; 14.52\left(\mathrm{CH}_{3}\right.$ from $\left.\mathrm{CO}_{2} \mathrm{Et}\right)$. Anal. calcd. for $\mathrm{C}_{23} \mathrm{H}_{20} \mathrm{~N}_{2} \mathrm{O}_{4}$ (388.42): $\mathrm{C}, 71.12$; H, 5.19; N, 7.21\%. Found: C, 71.06; H, 5.25; N, 7.16\%.

1-Carbethoxy-3-[(3-methoxyphenyl)carbamoyl]pyrrolo[2,1-a]isoquinoline (17). IR $\vee$ 3298, 3124, 1708, 1635, 1532. ${ }^{1} \mathrm{H}-\mathrm{NMR}(\delta \mathrm{ppm}, J \mathrm{~Hz}): 9.78(1 \mathrm{H}, \mathrm{m}, 10-\mathrm{H}) ; 9.38(1 \mathrm{H}, \mathrm{d}, 7.6,5-\mathrm{H})$; $8.01(1 \mathrm{H}, \mathrm{s}, \mathrm{NH}) ; 7.73(1 \mathrm{H}, \mathrm{s}, 2-\mathrm{H}) ; 7.70(1 \mathrm{H}, \mathrm{m}, 7-\mathrm{H}) ; 7.55-7.64(2 \mathrm{H}, \mathrm{m}, 8-\mathrm{H}, 9-\mathrm{H}) ; 7.28(1 \mathrm{H}, \mathrm{t}$, $8.1,16-\mathrm{H}) ; 7.13(1 \mathrm{H}, \mathrm{ddd}, 8.1,2.2,0.9,17-\mathrm{H}) ; 7.02(1 \mathrm{H}, \mathrm{d}, 7.6,6-\mathrm{H}) ; 6.72(1 \mathrm{H}, \mathrm{ddd}, 8.1,2.2$, $0.9,15-\mathrm{H}) ; 4.39\left(2 \mathrm{H}, \mathrm{q}, 7.1, \mathrm{CH}_{2}\right.$ from $\left.\mathrm{CO}_{2} \mathrm{Et}\right) ; 3.83(3 \mathrm{H}, \mathrm{s}, \mathrm{OMe}) ; 1.44\left(3 \mathrm{H}, \mathrm{t}, 7.1, \mathrm{CH}_{3}\right.$ from $\mathrm{CO}_{2} \mathrm{Et}$ ). ${ }^{13} \mathrm{C}-\mathrm{NMR} \delta 164.61$ (COO); 160.20 (14-C); 159.34 (11-C); 139.04 (12-C); 135.37 (10bC); 129.73 (16-C); 129.48 (6a-C); 128.68 (9-C or C-8); 127.53 (8-C or 9-C); 127.49 (10-C); 126.58 (7-C); 124.68 (10a-C); 124.32 (5-C); 119.84 (2-C); 118.81 (3-C); 114.62 (6-C); 112.34 (17-C); 110.23 (15-C); 108.76 (1-C); 10586 (13-C); $60.55\left(\mathrm{CH}_{2}\right.$ from $\left.\mathrm{CO}_{2} \mathrm{Et}\right) ; 55.32(\mathrm{OMe})$; $14.49\left(\mathrm{CH}_{3}\right.$ from $\left.\mathrm{CO}_{2} \mathrm{Et}\right)$. Anal. calcd. for $\mathrm{C}_{23} \mathrm{H}_{20} \mathrm{~N}_{2} \mathrm{O}_{4}$ (388.42): C, 71.12; H, 5.19; N, 7.21\%. Found: C, 71.19; H, 5.28; N, 7.16\%.

1-Carbethoxy-3-[(3-trifluoromethylphenyl)carbamoyl]pyrrolo[2,1-a]isoquinoline (18). IR $v$ 3320, 3126, 1703, 1669, 1532. ${ }^{1} \mathrm{H}-\mathrm{NMR}(\delta \mathrm{ppm}, J \mathrm{~Hz}): 9.42(1 \mathrm{H}, \mathrm{dd}, 7.8,2.2,10-\mathrm{H}) ; 9.11(1 \mathrm{H}$, d, 7.5, 5-H); $8.39(1 \mathrm{H}, \mathrm{s}, \mathrm{NH}) ; 7.89(1 \mathrm{H}, \mathrm{s}, 2-\mathrm{H}) ; 7.88(1 \mathrm{H}, \mathrm{bs}, 13-\mathrm{H}) ; 7.78(1 \mathrm{H}, \mathrm{dq}, 7.6,1.6,15-$ H); 7.55-7.64 (3H, m, 7-H, 8-H, 9-H); $7.52(1 \mathrm{H}, \mathrm{dd}, 8.0,7.6,16-\mathrm{H}) ; 7.46(1 \mathrm{H}, \mathrm{bd}, 8.0,17-\mathrm{H})$; $7.08(1 \mathrm{H}, \mathrm{d}, 7.5,6-\mathrm{H}) ; 4.47\left(2 \mathrm{H}, \mathrm{q}, 7.2, \mathrm{CH}_{2}\right.$ from $\left.\mathrm{CO}_{2} \mathrm{Et}\right) ; 1.47$ (3H, t, 7.2, $\mathrm{CH}_{3}$ from $\left.\mathrm{CO}_{2} \mathrm{Et}\right)$. ${ }^{13} \mathrm{C}-\mathrm{NMR} \quad \delta 166.10$ (COO); 160.27 (11-C); 137.46 (12-C); 135.98 (10b-C); 131.70 (14-C, q, $33.0) ; 129.81$ (8-C or 9-C); 129.80 (6a-C); 129.28 (C-9 or 8-C); 127.75 (7-C); 127.26 (10-C); 126.93 (16-C); $124.82\left(\mathrm{CF}_{3}, \mathrm{q}, 272.7\right) ; 124.24$ (10a-C); 124.12 (15-C, q, 1.2); 124.06 (5-C); 121.82 (17-C); 121.74 (2-C); 117.90 (3-C); 117.86 (13-C, q, 3.7); 115.43 (6-C); 108.63 (1-C); $61.76\left(\mathrm{CH}_{2}\right.$ from $\left.\mathrm{CO}_{2} \mathrm{Et}\right) ; 14.22\left(\mathrm{CH}_{3}\right.$ from $\left.\mathrm{CO}_{2} \mathrm{Et}\right)$. Anal. calcd. for $\mathrm{C}_{23} \mathrm{H}_{17} \mathrm{~F}_{3} \mathrm{~N}_{2} \mathrm{O}_{3}$ (426.39): $\mathrm{C}$, 64.79; H, 4.02; N, 6.57\%. Found: C, 64.76; H, 4.14; N, 6.63\%.

1,2-Dicarbomethoxy-3-[(2-methylphenyl)carbamoyl]pyrrolo[2,1-a]isoquinoline (19). IR $v$ 3319, 3070, 1714, 1654, 1523. ${ }^{1} \mathrm{H}-\mathrm{NMR}(\delta \mathrm{ppm}, J \mathrm{~Hz}): 10.21(1 \mathrm{H}, \mathrm{s}, \mathrm{NH}) ; 9.39(1 \mathrm{H}, \mathrm{d}, 7.7,5-$ $\mathrm{H}) ; 8.62(1 \mathrm{H}, \mathrm{m}, 10-\mathrm{H})$; $7.98(1 \mathrm{H}, \mathrm{d}, 8.0,17-\mathrm{H}) ; 7.69(1 \mathrm{H}, \mathrm{m}, 7-\mathrm{H})$; 7.54-7.60 (2H, m, 8-H, 9-H); 7.24-7.31 (2H, m, 14-H, 16-H); $7.12(1 \mathrm{H}, \mathrm{m}, 15-\mathrm{H}) ; 7.10(1 \mathrm{H}, \mathrm{d}, 7.7,6-\mathrm{H}) ; 4.01(3 \mathrm{H}, \mathrm{s}, 1-$ $\left.\mathrm{CO}_{2} \mathrm{Me}\right) ; 3.97\left(3 \mathrm{H}, \mathrm{s}, 2-\mathrm{CO}_{2} \mathrm{Me}\right) ; 2.41(3 \mathrm{H}, \mathrm{s}, 2-\mathrm{Me}) .{ }^{13} \mathrm{C}-\mathrm{NMR} \delta 167.42$ and 166.80 (COO); 158.77 (11-C); 135.69 (10b-C); 130.95 (6a-C); 130.71 (14-C); 130.31 (13-C); 128.98 (12-C); 128.73 (9-C or 8-C); 128.14 (8-C or 9-C); 127.13 (7-C); 126.49 (16-C); 125.41 (15-C); 124.96 (5-C); 124.76 (10-C); 124.22 (10a-C); 123.58 (17-C); 120.27 (2-C); 119.83 (3-C); 115.13 (6-C); 110.33 (1-C); 53.33 and $52.68\left(2 \mathrm{CH}_{3}\right.$ from 1,2- $\left.\mathrm{CO}_{2} \mathrm{Me}\right) ; 18.20$ (2-Me). Anal. calcd. for $\mathrm{C}_{24} \mathrm{H}_{20} \mathrm{~N}_{2} \mathrm{O}_{5}$ (416.43): C, 69.22; H, 4.84; N, 6.73\%. Found: C, 69.09; H, 4.90; N, 6.64\%.

1,2-Dicarbomethoxy-3-[(4-methylphenyl)carbamoyl]pyrrolo[2,1-a]isoquinoline (20). IR $\mathrm{v}$ 3310, 3025, 1726, 1699, 1663, 1544. ${ }^{1} \mathrm{H}-\mathrm{NMR}$ ( $\delta$ ppm, $\left.J \mathrm{~Hz}\right): 10.72(1 \mathrm{H}, \mathrm{s}, \mathrm{NH}) ; 9.42(1 \mathrm{H}, \mathrm{d}$, 7.8, 5-H); $8.56(1 \mathrm{H}, \mathrm{m}, 10-\mathrm{H}) ; 7.64(1 \mathrm{H}, \mathrm{m}, 7-\mathrm{H}) ; 7.63(2 \mathrm{H}, \mathrm{d}, 8.4,14-\mathrm{H}, 16-\mathrm{H}) ; 7.48-7.62(2 \mathrm{H}$, m, 8-H, 9-H); 7.19 (2H, d, 8.4, 13-H, 17-H); $7.17(1 \mathrm{H}, \mathrm{d}, 7.8,6-\mathrm{H}) ; 4.00\left(3 \mathrm{H}, \mathrm{s}, 1-\mathrm{CO}_{2} \mathrm{Me}\right) ; 3.97$ $\left(3 \mathrm{H}, \mathrm{s}, 2-\mathrm{CO}_{2} \mathrm{Me}\right) ; 2.35$ (3H, s, 4-Me). ${ }^{13} \mathrm{C}-\mathrm{NMR} \delta 167.55$ and 166.92 (COO); 158.15 (11-C); 
135.56 (10b-C); 134.04 (12-C); 130.54 (15-C); 129.52 (10-C); 129.49 (13-C); 128.85 (6a-C); 128.59 (8-C or 9-C); 128.07 (9-C or 8-C); 127.09 (7-C); 124.82 (5-C); 124.12 (10a-C); 120.60 (2-C); 120.18 (14-C, 16-C); 119.12 (3-C); 115.04 (6-C); 110.54 (1-C); 53.30, 52.64 (2 $\mathrm{CH}_{3}$ from 1,2- $\mathrm{CO}_{2} \mathrm{Me}$ ); 20.91 (4-Me). Anal. calcd. for $\mathrm{C}_{24} \mathrm{H}_{20} \mathrm{~N}_{2} \mathrm{O}_{5}$ (416.43): C, 69.22; H, 4.84; N, 6.73\%. Found: C, 69.26; H, 4.78; N, 6.79\%.

\section{1,2-Dicarbomethoxy-3-[(3-trifluoromethylphenyl)carbamoyl]pyrrolo[2,1-a]isoquinoline}

(21). IR v 3154, 3022, 1718, 1691, 1664, 1576. ${ }^{1} \mathrm{H}-\mathrm{NMR}(\delta \mathrm{ppm}, J \mathrm{~Hz}): 11.28(1 \mathrm{H}, \mathrm{s}, \mathrm{NH}) ; 9.47$ $(1 \mathrm{H}, \mathrm{d}, 7.8,5-\mathrm{H}) ; 8.43(1 \mathrm{H}, \mathrm{m}, 10-\mathrm{H}) ; 8.16(1 \mathrm{H}, \mathrm{bt}, 1.8,13-\mathrm{H}) ; 7.88(1 \mathrm{H}, \mathrm{bd}, 7.9,15-\mathrm{H}) ; 7.68$ $(1 \mathrm{H}, \mathrm{m}, 7-\mathrm{H}) ; 7.53-7.62(2 \mathrm{H}, \mathrm{m}, 8-\mathrm{H}, 9-\mathrm{H}) ; 7.49(1 \mathrm{H}, \mathrm{t}, 7.9,16-\mathrm{H}) ; 7.40(1 \mathrm{H}, \mathrm{bd}, 7.9,17-\mathrm{H})$; $7.10(1 \mathrm{H}, \mathrm{d}, 7.8,6-\mathrm{H}) ; 4.03\left(3 \mathrm{H}, \mathrm{s}, 1-\mathrm{CO}_{2} \mathrm{Me}\right) ; 4.00\left(3 \mathrm{H}, \mathrm{s}, 2-\mathrm{CO}_{2} \mathrm{Me}\right) .{ }^{13} \mathrm{C}-\mathrm{NMR} \delta 167.65$ and 167.07 (COO); 158.57 (11-C); 138.14 (12-C); 131.44 (14-C, q, 32.2); 130.67 (10b-C); 129.47 (7C); 128.87 (6a-C); 128.81 (8-C or 9-C); 128.30 (9-C or 8-C); 127.22 (10-C); 124.80 (16-C); 124.36 (5-C); 124.05 (10a-C); $123.94\left(\mathrm{CF}_{3}, \mathrm{q}, 272.6\right) ; 123.21$ (17-C); 120.83 (15-C, q, 3.8); 120.00 (2-C); 119.00 (3-C); 115.46 (6-C); 111.27 (1-C); 53.81, $53.56\left(2 \mathrm{CH}_{3}\right.$ from 1,2- $\left.\mathrm{CO}_{2} \mathrm{Me}\right)$. Anal. calcd. for $\mathrm{C}_{24} \mathrm{H}_{17} \mathrm{~F}_{3} \mathrm{~N}_{2} \mathrm{O}_{5}$ (470.40): C, 61.28; H, 3.64; N, 5.95\%. Found: C, 61.34; H, 3.70; N, $6.05 \%$.

1-Carbethoxy-3-[(3-chloro-4-fluorophenyl)carbamoyl]pyrrolo[2,1-a]isoquinoline (22). IR v 3339, 3125, 1700, 1684, 1660, 1536. ${ }^{1} \mathrm{H}-\mathrm{NMR}(\delta \mathrm{ppm}, J \mathrm{~Hz}): 9.74(1 \mathrm{H}, \mathrm{m}, 10-\mathrm{H}) ; 9.30(1 \mathrm{H}, \mathrm{d}$, 7.6, 5-H); $7.86(1 \mathrm{H}, \mathrm{s}, \mathrm{NH}) ; 7.83(1 \mathrm{H}, \mathrm{dd}, 6.6,2.2,13-\mathrm{H}) ; 7.77(1 \mathrm{H}, \mathrm{s}, 2-\mathrm{H}) ; 7.68(1 \mathrm{H}, \mathrm{m}, 7-\mathrm{H})$; 7.55-7.61 (2H, m, 8-H, 9-H); $7.44(1 \mathrm{H}, \mathrm{ddd}, 8.7,4.0,2.2,17-\mathrm{H}) ; 7.14(1 \mathrm{H}, \mathrm{t}, 8.7,16-\mathrm{H}) ; 7.11$ $(1 \mathrm{H}, \mathrm{d}, 7.6,6-\mathrm{H}) ; 4.43\left(2 \mathrm{H}, \mathrm{q}, 7.1, \mathrm{CH}_{2}\right.$ from $\left.\mathrm{CO}_{2} \mathrm{Et}\right) ; 1.46\left(3 \mathrm{H}, \mathrm{t}, 7.1, \mathrm{CH}_{3}\right.$ from $\left.\mathrm{CO}_{2} \mathrm{Et}\right) .{ }^{13} \mathrm{C}-$ NMR $\delta 164.61$ (COO); 159.25 (11-C); 154.91 (15-C, d, 246.2); 135.68 (10b-C); 134.49 (12-C, d, $3.3) ; 129.63$ (6a-C); 128.91 (8-C or 9-C); 127.65 (9-C or 8-C); 127.64 (10-C); 126.70 (7-C); 124.73 (10a-C); 124.33 (5-C); 122.52 (13-C); 121.32 (14-C, d, 18.2); 120.05 (2-C); 119.92 (17$\mathrm{C}, \mathrm{d}, 6.8) ; 118.26$ (3-C); 116.70 (16-C, d, 22.1); 114.92 (6-C); 109.08 (1-C); $60.67\left(\mathrm{CH}_{2}\right.$ from $\left.\mathrm{CO}_{2} \mathrm{Et}\right) ; 14.54\left(\mathrm{CH}_{3}\right.$ from $\left.\mathrm{CO}_{2} \mathrm{Et}\right)$. Anal. calcd. for $\mathrm{C}_{22} \mathrm{H}_{16} \mathrm{ClFN}_{2} \mathrm{O}_{3}$ (410.83): C, 64.32; $\mathrm{H}, 3.92$; N, 6.82\%. Found: C, 64.26; H, 4.11; N, 6.86\%.

1,2-Dicarbomethoxy-3-[(4-chlorophenyl)carbamoyl]pyrrolo[2,1-a]isoquinoline (23). IR $v$ 3277, 3166, 1731, 1703, 1651, 1557. ${ }^{1} \mathrm{H}-\mathrm{NMR}(\delta \mathrm{ppm}, J \mathrm{~Hz}): 11.05(1 \mathrm{H}, \mathrm{s}, \mathrm{NH}) ; 9.42(1 \mathrm{H}, \mathrm{d}$, 7.8, 5-H); $8.46(1 \mathrm{H}, \mathrm{m}, 10-\mathrm{H}) ; 7.71(2 \mathrm{H}, \mathrm{d}, 8.8,13-\mathrm{H}, 17-\mathrm{H}) ; 7.68(1 \mathrm{H}, \mathrm{m}, 7-\mathrm{H}) ; 7.52-7.62(2 \mathrm{H}$, m, 8-H, 9-H); $7.32(2 \mathrm{H}, \mathrm{d}, 8.8,14-\mathrm{H}, 16-\mathrm{H}) ; 7.09(1 \mathrm{H}, \mathrm{d}, 7.8,6-\mathrm{H}) ; 4.02\left(\mathrm{~s}, \mathrm{CH}_{3}\right.$ from 1$\left.\mathrm{CO}_{2} \mathrm{Me}\right) ; 3.98\left(\mathrm{~s}, \mathrm{CH}_{3}\right.$ from $\left.2-\mathrm{CO}_{2} \mathrm{Me}\right) .{ }^{13} \mathrm{C}-\mathrm{NMR} \delta 167.65$ and 167.01 (COO); 158.32 (11-C); 136.84 (10b-C); 130.61 (12-C); 129.30 (6a-C); 129.02 (14-C, 16-C); 128.87 (15-C); 128.74 (9-C or 8-C); 128.24 (8-C or 9-C); 127.18 (7-C); 124.80 (10-C); 124.43 (5-C); 124.11 (10a-C); 121.41 (13-C, 17-C); 120.43 (2-C); 119.00 (3-C); 115.32 (6-C); 111.01 (1-C); 53.44, 52.74 (2 $\mathrm{CH}_{3}$ from 1,2- $\mathrm{CO}_{2} \mathrm{Me}$ ). Anal. calcd. for $\mathrm{C}_{23} \mathrm{H}_{17} \mathrm{ClN}_{2} \mathrm{O}_{5}$ (436.85): C, 63.24; H, 3.92; N, 6.41\%. Found: $\mathrm{C}$, 63.32; H, 4.02; N, 6.53\%.

1,2-Dicarbomethoxy-3-[(2,6-dimethylphenyl)carbamoyl]pyrrolo[2,1-a]isoquinoline (24). IR v 3367, 3142, 1740, 1698, 1653, 1540. ${ }^{1} \mathrm{H}-\mathrm{NMR}(\delta \mathrm{ppm}, J \mathrm{~Hz}): 10.02(1 \mathrm{H}, \mathrm{s}, \mathrm{NH}) ; 9.47(1 \mathrm{H}, \mathrm{d}$, 7.7, 5-H); $8.46(1 \mathrm{H}, \mathrm{m}, 10-\mathrm{H}) ; 7.68(1 \mathrm{H}, \mathrm{m}, 7-\mathrm{H})$; 7.52-7.62 (2H, m, 8-H, 9-H); 7.15 (3H, bs, 14$\mathrm{H}, 15-\mathrm{H}, 16-\mathrm{H}) ; 7.09(1 \mathrm{H}, \mathrm{d}, 7.8,6-\mathrm{H}) ; 4.02\left(3 \mathrm{H}, \mathrm{s}, \mathrm{CH}_{3}\right.$ from $\left.1-\mathrm{CO}_{2} \mathrm{Me}\right) ; 3.98\left(3 \mathrm{H}, \mathrm{s}, \mathrm{CH}_{3}\right.$ from 2- $\left.\mathrm{CO}_{2} \mathrm{Me}\right) ; 2.31$ (6H, s, $\left.2 \mathrm{CH}_{3} 2,6-\mathrm{diMe}\right) .{ }^{13} \mathrm{C}-\mathrm{NMR} \delta 167.62$ and 166.83 (COO); 158.84 (11-C); 
135.42 (13-C, 17-C); 133.73 (10b-C); 130.84 (12-C); 128.95 (6a-C); 128.68 (8-C or 9-C); 128.21 (14-C, 16-C); 128.12 (9-C or 8-C); 127.31 (15-C); 127.10 (7-C); 125.00 (5-C); 124.70 (10-C); 124.19 (10a-C); 119.85 (2-C); 119.83 (3-C); 115.23 (6-C); 110.38 (1-C); 53.30, $52.66\left(2 \mathrm{CH}_{3}\right.$ from 1,2- $\left.\mathrm{CO}_{2} \mathrm{Me}\right) ; 18.59\left(2 \mathrm{CH}_{3}\right.$ from 2,6-diMe). Anal. calcd. for $\mathrm{C}_{25} \mathrm{H}_{22} \mathrm{~N}_{2} \mathrm{O}_{5}$ (430.46): C, 69.76; H, 5.15; N, 6.51\%. Found: C, 69.86; H, 5.25; N, 6.56\%.

1,2-Dicarbomethoxy-3-[(2,6-diethylphenyl)carbamoyl]pyrrolo[2,1-a]isoquinoline (25). IR $v$ 3269, 3143, 1725, 1696, 1650, 1538. ${ }^{1} \mathrm{H}-\mathrm{NMR}(\delta \mathrm{ppm}, J \mathrm{~Hz}): 10.04(1 \mathrm{H}, \mathrm{s}, \mathrm{NH}) ; 9.50(1 \mathrm{H}, \mathrm{d}$, 7.7, 5-H); $8.61(1 \mathrm{H}, \mathrm{m}, 10-\mathrm{H}) ; 7.68(1 \mathrm{H}, \mathrm{m}, 7-\mathrm{H}) ; 7.54-7.62(2 \mathrm{H}, \mathrm{m}, 8-\mathrm{H}, 9-\mathrm{H}) ; 7.28(1 \mathrm{H}, \mathrm{t}, 7.8$, $15-\mathrm{H}) ; 7.19(2 \mathrm{H}, \mathrm{d}, 7.8,14-\mathrm{H}, 16-\mathrm{H}) ; 7.10(1 \mathrm{H}, \mathrm{d}, 7.7,6-\mathrm{H}) ; 4.03\left(3 \mathrm{H}, \mathrm{s}, \mathrm{CH}_{3}\right.$ from $\left.1-\mathrm{CO}_{2} \mathrm{Me}\right)$; $3.98\left(3 \mathrm{H}, \mathrm{s}, \mathrm{CH}_{3}\right.$ from $\left.2-\mathrm{CO}_{2} \mathrm{Me}\right) ; 2.66\left(4 \mathrm{H}, \mathrm{q}, 7.6,2 \mathrm{CH}_{2}\right.$ from 2,6-diEt); $1.21\left(6 \mathrm{H}, \mathrm{t}, 7.6,2 \mathrm{CH}_{3}\right.$ from 2,6-diEt). ${ }^{13} \mathrm{C}-\mathrm{NMR} \delta 167.72$ and 166.86 (COO); 159.59 (11-C); 141.54 (13-C, 17-C); 132.38 (10b-C); 130.86 (6a-C); 128.99 (12-C); 128.71 (8-C or 9-C); 128.13 (9-C or 8-C); 127.95 (15-C); 127.12 (7-C); 126.46 (14-C, 16-C); 125.09 (5-C); 124.70 (10-C); 124.20 (10a-C); 119.88 (3-C); 119.76 (2-C); 115.26 (6-C); 110.43 (1-C); 53.30, $52.69\left(2 \mathrm{CH}_{3}\right.$ from 1,2- $\left.\mathrm{CO}_{2} \mathrm{Me}\right) ; 25.06$ $\left(2 \mathrm{CH}_{2}\right.$ 2,6-diEt); 14.59 (2 $\mathrm{CH}_{3}$ from 2,6-diEt). Anal. calcd. for $\mathrm{C}_{27} \mathrm{H}_{26} \mathrm{~N}_{2} \mathrm{O}_{5}$ (458.51): C, 70.73; H, $5.71 ; \mathrm{N}, 6.11 \%$. Found: C, 70.75; H, 5.66; N, 6.14\%.

\section{1,2-Dicarbomethoxy-3-[(2-methyl-3-chlorophenyl)carbamoyl]pyrrolo[2,1-a]isoquinoline}

(26). IR $v 3154,3022,1718,1691,1664,1576 .{ }^{1} \mathrm{H}-\mathrm{NMR}(\delta \mathrm{ppm}, J \mathrm{~Hz}): 11.12(1 \mathrm{H}, \mathrm{s}, \mathrm{NH}) ; 9.32$ $(1 \mathrm{H}, \mathrm{d}, 7.5,5-\mathrm{H}) ; 8.28(1 \mathrm{H}, \mathrm{m}, 10-\mathrm{H}) ; 7.74(1 \mathrm{H}, \mathrm{m}, 7-\mathrm{H}) ; 7.57-7.68(2 \mathrm{H}, \mathrm{m}, 8-\mathrm{H}, 9-\mathrm{H}) ; 7.38(1 \mathrm{H}$, $\mathrm{dd}, 8.0,1.8,15-\mathrm{H}) ; 7.37(1 \mathrm{H}, \mathrm{dd}, 8.0,1.8,17-\mathrm{H}) ; 7.20(1 \mathrm{H}, \mathrm{t}, 8.0,16-\mathrm{H}) ; 7.19(1 \mathrm{H}, \mathrm{d}, 7.5,6-\mathrm{H})$; $4.12\left(3 \mathrm{H}, \mathrm{s}, \mathrm{CH}_{3}\right.$ from 1- $\left.\mathrm{CO}_{2} \mathrm{Me}\right) ; 4.02\left(3 \mathrm{H}, \mathrm{s}, \mathrm{CH}_{3}\right.$ from $\left.2-\mathrm{CO}_{2} \mathrm{Me}\right) ; 2.40(3 \mathrm{H}, \mathrm{s}, 2-\mathrm{Me}) .{ }^{13} \mathrm{C}-$ NMR $\delta 169.25$ and 167.19 (COO); 160.44 (11-C); 135.72 (10b-C); 135.11 (12-C); 132.25 (13C); 131.67 (14-C); 129.41 (8-C or 9-C); 129.11 (6a-C); 128.72 (15-C); 128.58 (9-C or 8-C); 127.59 (7-C); 127.07 (16-C); 124.79 (17-C); 124.66 (5-C); 124.12 (10-C); 123.73 (10a-C); 120.05 (3-C); 118.84 (2-C); 116.32 (6-C); 110.93 (1-C); 53.76, $53.69\left(2 \mathrm{CH}_{3}\right.$ from 1,2- $\left.\mathrm{CO}_{2} \mathrm{Me}\right)$; $15.04\left(2-\mathrm{CH}_{3}\right)$. Anal. calcd. for $\mathrm{C}_{24} \mathrm{H}_{19} \mathrm{ClN}_{2} \mathrm{O}_{5}$ (450.88): C, 63.93; H, 4.25; N, 6.21\%. Found: $\mathrm{C}$, $64.05 ; \mathrm{H}, 4.15 ; \mathrm{N}, 6.14 \%$.

\section{1,2-Dicarbomethoxy-3-[(2-methyl-4-chlorophenyl)carbamoyl]pyrrolo[2,1-a]isoquinoline} (27). IR 3252, 3037, 1700, 1696, 1663, 1543. ${ }^{1} \mathrm{H}-\mathrm{NMR}(\delta \mathrm{ppm}, J \mathrm{~Hz}): 10.87$ (1H, s, NH); 9.31 $(1 \mathrm{H}, \mathrm{d}, 7.7,5-\mathrm{H}) ; 8.37(1 \mathrm{H}, \mathrm{m}, 10-\mathrm{H}) ; 7.72(1 \mathrm{H}, \mathrm{m}, 7-\mathrm{H}) ; 7.52-7.63(2 \mathrm{H}, \mathrm{m}, 8-\mathrm{H}, 9-\mathrm{H}) ; 7.46(1 \mathrm{H}$, d, 8.5, 17-H); $7.27(1 \mathrm{H}, \mathrm{d}, 2.5,14-\mathrm{H}) ; 7.25(1 \mathrm{H}, \mathrm{dd}, 8.5,2.5,16-\mathrm{H}) ; 4.08\left(3 \mathrm{H}, \mathrm{s}, \mathrm{CH}_{3}\right.$ from 1$\left.\mathrm{CO}_{2} \mathrm{Me}\right) ; 3.99\left(3 \mathrm{H}, \mathrm{s}, \mathrm{CH}_{3}\right.$ from 2-CO $\left.\mathrm{CO}_{2} \mathrm{Me}\right) ; 2.35(3 \mathrm{H}, \mathrm{s}, 2-\mathrm{Me}) .{ }^{13} \mathrm{C}-\mathrm{NMR} \delta 167.91$ and 167.25 (COO), 159.32 (11-C); 134.12 (10b-C); 133.19 (12-C); 131.13 (13-C); 130.76 (14-C); 129.15 (8C or 9-C); 129.07 (6a-C); 128.51 (9-C or 8-C); 127.40 (7-C, 16-C); 126.32 (17-C); 124.78 (5-C); 124.40 (10-C); 123.98 (10a-C); 120.16 (3-C); 119.99 (2-C); 115.91 (6-C); 111.07 (1-C); 53.55, $53.17\left(2 \mathrm{CH}_{3}\right.$ from 1,2- $\left.\mathrm{CO}_{2} \mathrm{Me}\right) ; 17.92\left(2-\mathrm{CH}_{3}\right)$. Anal. calcd. for $\mathrm{C}_{24} \mathrm{H}_{19} \mathrm{ClN}_{2} \mathrm{O}_{5}$ (450.88): C, $63.93 ; \mathrm{H}, 4.25 ; \mathrm{N}, 6.21 \%$. Found: C, 63.89; H, 4.20; N, 6.29\%.

\section{1,2-Dicarbomethoxy-3-[(2-methyl-5-chlorophenyl)carbamoyl]pyrrolo[2,1-a]isoquinoline}

(28). IR $v 3289,3001,1712,1696,1653,1579 .{ }^{1} \mathrm{H}-\mathrm{NMR}(\delta \mathrm{ppm}, J \mathrm{~Hz}): 10.37(1 \mathrm{H}, \mathrm{s}, \mathrm{NH}) ; 9.41$ $(1 \mathrm{H}, \mathrm{d}, 7.7,5-\mathrm{H}) ; 8.58(1 \mathrm{H}, \mathrm{m}, 10-\mathrm{H}) ; 8.15(1 \mathrm{H}, \mathrm{d}, 2.1,17-\mathrm{H}) ; 7.70(1 \mathrm{H}, \mathrm{m}, 7-\mathrm{H}) ; 7.52-7.62(2 \mathrm{H}$, m, 8-H, 9-H); $7.13(1 \mathrm{H}, \mathrm{d}, 8.1,14-\mathrm{H}) ; 7.10(1 \mathrm{H}, \mathrm{d}, 7.7,6-\mathrm{H}) ; 7.09(1 \mathrm{H}, \mathrm{dd}, 8.1,2.1,15-\mathrm{H}) ; 4.02$ (3H, s, $\mathrm{CH}_{3}$ from 1- $\left.\mathrm{CO}_{2} \mathrm{Me}\right) ; 3.97$ (3H, s, $\mathrm{CH}_{3}$ from 2- $\left.\mathrm{CO}_{2} \mathrm{Me}\right) ; 2.39$ (3H, s, 2-Me). ${ }^{13} \mathrm{C}-\mathrm{NMR} \delta$ 
167.40 and 166.75 (COO); 158.77 (11-C); 136.94 (10b-C); 131.86 (12-C); 131.07 (13-C); 129.06 (6a-C); 129.05 (16-C); 128.80 (8-C or 9-C); 128.22 (9-C or 8-C); 127.17 (7-C); 125.09 (15-C); 124.97 (5-C, 14-C); 124.73 (10-C); 124.26 (10a-C); 123.26 (17-C); 120.08 (2-C); 119.74 (3-C); 115.29 (6-C); 110.73 (1-C); 53.30, $52.61\left(2 \mathrm{CH}_{3}\right.$ from 1,2- $\left.\mathrm{CO}_{2} \mathrm{Me}\right) ; 17.76\left(\mathrm{CH}_{3}\right.$ from 2$\mathrm{Me}$ ). Anal. calcd. for $\mathrm{C}_{24} \mathrm{H}_{19} \mathrm{ClN}_{2} \mathrm{O}_{5}$ (450.88): C, 63.93; H, 4.25; N, 6.21\%. Found: C, 63.88; H, $4.32 ; \mathrm{N}, 6.18 \%$.

\section{1,2-Dicarbomethoxy-3-[(3,4-methylenedioxyphenyl)carbamoyl]pyrrolo-[2,1-a]isoquinoline} (29). IR 3248, 3027, 1732, 1701, 1650, 1536. ${ }^{1} \mathrm{H}-\mathrm{NMR}(\delta \mathrm{ppm}, J \mathrm{~Hz}): 10.81(1 \mathrm{H}, \mathrm{s}, \mathrm{NH}) ; 9.42$ $(1 \mathrm{H}, \mathrm{d}, 7.8,5-\mathrm{H}) ; 8.51(1 \mathrm{H}, \mathrm{m}, 10-\mathrm{H}) ; 7.68(1 \mathrm{H}, \mathrm{m}, 7-\mathrm{H}) ; 7.52-7.62(2 \mathrm{H}, \mathrm{m}, 8-\mathrm{H}, 9-\mathrm{H}) ; 7.48(1 \mathrm{H}$, d, 2.1, 13-H); $7.10(1 \mathrm{H}, \mathrm{d}, 7.8,6-\mathrm{H}) ; 7.09(1 \mathrm{H}, \mathrm{dd}, 8.4,2.1,17-\mathrm{H}) ; 6.80(1 \mathrm{H}, \mathrm{d}, 8.4,16-\mathrm{H}) ; 5.98$ $\left(2 \mathrm{H}, \mathrm{s}, \mathrm{CH}_{2}\right) ; 4.01\left(3 \mathrm{H}, \mathrm{s}, \mathrm{CH}_{3}\right.$ from 1- $\left.\mathrm{CO}_{2} \mathrm{Me}\right) ; 3.98\left(3 \mathrm{H}, \mathrm{s}, \mathrm{CH}_{3}\right.$ from $\left.2-\mathrm{CO}_{2} \mathrm{Me}\right) .{ }^{13} \mathrm{C}-\mathrm{NMR} \delta$ 167.65 and 166.95 (COO); 158.16 (11-C); 147.89 (14-C); 144.41 (15-C); 132.56 (10b-C); 130.63 (12-C); 128.98 (6a-C); 128.66 (8-C or 9-C); 128.16 (9-C or 8-C); 127.16 (7-C); 124.96 (5-C); 124.60 (10-C); 124.30 (10a-C); 120.69 (2-C); 119.08 (3-C); 115.14 (6-C); 113.44 (17-C); 110.84 (1-C); 108.19 (16-C); $102.86(13-\mathrm{C}) ; 101.25\left(\mathrm{CH}_{2}\right) ; 53.29,52.60\left(2 \mathrm{CH}_{3}\right.$ from 1,2$\mathrm{CO}_{2} \mathrm{Me}$ ). Anal. calcd. for $\mathrm{C}_{24} \mathrm{H}_{18} \mathrm{~N}_{2} \mathrm{O}_{7}$ (446.42): C, 64.57; H, 4.06; N, 6.27\%. Found: C, 64.64; $\mathrm{H}, 4.15 ; \mathrm{N}, 6.32 \%$.

\section{General procedure for carbamoyl substituted indolizine 30 and pyrrolo[2,1-a]isoquinolines} 31-32

(a) A solution of $\mathrm{N}$-methylcarbamoyl quaternary salt $(10 \mathrm{mmol}$ ), olefine (acrylonitrile or crotononitrile, $40 \mathrm{mmol})$, TPCD $(4.0 \mathrm{~g}, 6.5 \mathrm{mmol})$ and pyridine $(2.0 \mathrm{~mL})$ in DMF $(40 \mathrm{~mL})$ was stirred at $90{ }^{\circ} \mathrm{C}$ for $2 \mathrm{~h}$. The mixture was then cooled to room temperature and poured into $5 \%$ aq. $\mathrm{HCl}(100 \mathrm{~mL})$. The solution was extracted with chloroform $(4 \times 50 \mathrm{~mL})$ and the combined extracts were washed with water $(2 \times 50 \mathrm{~mL})$, dried $\left(\mathrm{Na}_{2} \mathrm{SO}_{4}\right)$ and evaporated to give a solid compound. This was purified by recrystallisation.

(b) The olefinic compound $(40 \mathrm{mmol})$ was added at room temperature to a stirred mixture of $\mathrm{N}$-methylcarbamoyl quaternary salt $(10 \mathrm{mmol})$ and TPCD $(4.0 \mathrm{~g}, 6.5 \mathrm{mmol})$ in 1,2-epoxybutane $(50 \mathrm{~mL})$. The reaction mixture was heated to reflux for 5-8 h, then it was concentrated under reduced pressure. The residue was cooled to room temperature and then was treated with $5 \%$ aq. $\mathrm{HCl}(100 \mathrm{~mL})$ and was worked up as described above.

The yields and m. p. for compounds 30-32 are shown in Table 4; the spectral data are given below.

1-Cyano-2,7-dimethyl-3-[(3,4-methylenedioxyphenyl)carbamoyl]indolizine (30). IR $\vee 3356$, 3053, 2205, 1636, 1536. ${ }^{1} \mathrm{H}-\mathrm{NMR}$ ( $\left.\delta \mathrm{ppm}, J \mathrm{~Hz}\right): 9.09(1 \mathrm{H}, \mathrm{d}, 7.2,5-\mathrm{H}) ; 7.82(1 \mathrm{H}, \mathrm{s}, \mathrm{NH}) ; 7.40$ $(1 \mathrm{H}, \mathrm{bs}, \mathrm{H}-8) ; 6.98(1 \mathrm{H}, \mathrm{bs}, 11-\mathrm{H}) ; 6.82(2 \mathrm{H}, \mathrm{s}, 14-\mathrm{H}, 15-\mathrm{H}) ; 6.84(1 \mathrm{H}, \mathrm{dd}, 7.2,1.8,6-\mathrm{H}) ; 6.00$ $\left(2 \mathrm{H}, \mathrm{s}, \mathrm{CH}_{2}\right) ; 2.69\left(3 \mathrm{H}, \mathrm{s}, 7-\mathrm{CH}_{3}\right) ; 2.45\left(3 \mathrm{H}, \mathrm{s}, 2-\mathrm{CH}_{3}\right) .{ }^{13} \mathrm{C}-\mathrm{NMR} \delta 161.86(9-\mathrm{C}) ; 148.23(12-\mathrm{C})$; 146.53 (13-C); 140.75 (8a-C); 139.44 (10-C); 133.74 (3-C); 128.89 (7-C); 127.92 (5-C); 117.75 (6-C); 117.25 (14-C or 15-C); 115.57 (8-C); 115.08 (2-C or CN); 114.97 (CN or 2-C); 108.54 (15-C or 14-C); 105.47 (11-C); $101.81\left(\mathrm{CH}_{2}\right) ; 83.06(1-\mathrm{C}) ; 21.06\left(7-\mathrm{CH}_{3}\right), 12.96\left(2-\mathrm{CH}_{3}\right)$. Anal. calcd. for $\mathrm{C}_{19} \mathrm{H}_{15} \mathrm{~N}_{3} \mathrm{O}_{3}$ (333.35): C, 68.46; H, 4.54; N, 12.60\%. Found: C, 68.26; H, 4.49; N, $12.68 \%$. 
1-Cyano-2-methyl-3-[(3-trifluoromethylphenyl)carbamoyl]pyrrolo[2,1-a]isoquinoline (31). IR $v 3297,3073,2204,1669,1541 .{ }^{1} \mathrm{H}-\mathrm{NMR}(\delta \mathrm{ppm}, J \mathrm{~Hz}): 8.71(1 \mathrm{H}, \mathrm{d}, 7.6,5-\mathrm{H}) ; 8.60(1 \mathrm{H}, \mathrm{m}$, $10-\mathrm{H}) ; 8.11(1 \mathrm{H}, \mathrm{s}, \mathrm{NH}) ; 8.05(1 \mathrm{H}, \mathrm{bs}, 13-\mathrm{H}) ; 7.82(1 \mathrm{H}, \mathrm{bd}, 8.1,17-\mathrm{H}) ; 7.49-7.62(4 \mathrm{H}, \mathrm{m}, 7-\mathrm{H}$, 8-H, 9-H, 16-H); 7.48 (1H, bd, 7.9, 15-H); $6.82(1 \mathrm{H}, \mathrm{d}, 7.6,6-\mathrm{H}) ; 2.75\left(3 \mathrm{H}, \mathrm{s}, 2-\mathrm{CH}_{3}\right) .{ }^{13} \mathrm{C}-\mathrm{NMR}$ ठ 159.24 (11-C); 139.04 (12-C); 135.26 (10b-C); 131.70 (14-C, q, 32.3); 129.78 (16-C); 129.69 (6a-C); 129.29 (8-C or 9-C); 128.41 (9-C or 8-C); 127.11 (7-C); 125.60 (10a-C); 123.80 (5-C); 123.13 (10-C, 17-C); 122.83 (2-C); 121.40 (15-C, q, 3.7); 119.40 ( $\left.\mathrm{CF}_{3}, 271.4\right) ; 118.60$ (3-C); $117.47(\mathrm{CN}) ; 116.88$ (13-C, q, 3.7); 114.04 (6-C); 86.42 (1-C); 12.69 (2- $\left.\mathrm{CH}_{3}\right)$. Anal. calcd. for $\mathrm{C}_{229} \mathrm{H}_{14} \mathrm{~F}_{3} \mathrm{~N}_{3} \mathrm{O}$ (393.37): C, 67.17; H, 3.59; N, 10.68\%. Found: C, 67.21; H, 3.64; N, 10.48\%.

1-Cyano-3-[(3-methoxyphenyl)carbamoyl]pyrrolo[2,1-a]isoquinoline (32). IR $v$ 3363, 3130, 2213, 1658, 1547. ${ }^{1} \mathrm{H}-\mathrm{NMR}(\delta \mathrm{ppm}, J \mathrm{~Hz}): 9.16(1 \mathrm{H}, \mathrm{d}, 7.6,5-\mathrm{H}) ; 8.73(1 \mathrm{H}, \mathrm{m}, 10-\mathrm{H}) ; 8.33(1 \mathrm{H}$, bs, NH); 7.69-7.79 (3H, m, 7-H, 8-H, 9-H); $7.72(1 \mathrm{H}, \mathrm{s}, 2-\mathrm{H}) ; 7.35(1 \mathrm{H}, \mathrm{t}, 8.1,16-\mathrm{H}) ; 7.23(1 \mathrm{H}$, d, 7.6, 6-H); $7.15(1 \mathrm{H}, \mathrm{t}, 2.3,13-\mathrm{H}) ; 7.06(1 \mathrm{H}, \mathrm{dd}, 8.1,2.3,17-\mathrm{H}) ; 6.86(1 \mathrm{H}, \mathrm{dd}, 8.1,2.3,15-\mathrm{H})$; $3.88\left(3 \mathrm{H}, \mathrm{s}, \mathrm{CH}_{3}\right) .{ }^{13} \mathrm{C}-\mathrm{NMR} \delta 159.70(11-\mathrm{C}) ; 159.60$ (14-C); 138.15 (12-C); 136.74 (10b-C); 130.40 (16-C); 130.27 (8-C or 9-C); 129.17 (6a-C); 129.07 (C-9 or C-8); 124.33 (10-C); 124.28 (10a-C); 123.37 (5-C); 121.16 (2-C); 119.36 (3-C); 116.21 (CN); 115.92 (6-C); 115.40 (17-C); 112.26 (15-C); 108.85 (13-C); 83.21 (1-C); $55.71\left(\mathrm{CH}_{3}\right)$. Anal. calcd. for $\mathrm{C}_{21} \mathrm{H}_{15} \mathrm{~N}_{3} \mathrm{O}_{2}(341.37)$ : C, 73.89; H, 4.43; N, 12.31\%. Found: C, 73.93; H, 4.53; N, 12.38\%.

\section{Acknowledgements}

S.C. Oltchim S.A., Romania, supported this work, in part.

\section{References}

1. Katritzky, A. R.; Rees C. W. Comprehensive Heterocyclic Chemistry, Potts, K. T., Ed.; Pergamon Press: Oxford, New York; 1984.

2. Hermecz, I.; Meszaros M. Adv. Heterocyclic Chem. 1983, 33, 241.

3. Huisgen, R. Angew. Chem., Int. Ed. 1963, 2, 565.

4. Zugrăvescu, I.; Petrovanu, M. N-Ylides Chemistry, McGraw-Hill Int.: New York, 1976.

5. Zugrăvescu, I.; Petrovanu, M, Eds.; Cicloadiții 3+2 dipolare, Academiei: Bucureşti, 1987.

6. Huisgen, R.; Grashey, R.;. Steingruber, E. Tetrahedron Lett. 1963, 1441.

7. Hendrick, C. A.; Ritchie, E.; Taylor, W. C. Aust. J. Chem. 1967, 20, 2467.

8. Basketter, N. S.; Plunkett, A. O. J. Chem. Soc.,Chem. Commun. 1973, 188.

9. Kakei, A.; Ito, S. Bull. Chem. Soc. Jpn. 1974, 47, 938.

10. Frohlich, J.; Kronke, F. Chem. Ber. 1971, 104, 1621.

11. Wei, X.; Hu, Y.; Li, T.; Hu, H. J. Chem. Soc., Perkin Trans. 1 1993, 20, 2487.

12. Zhou, J.; Hu, Y.; Hu, H. J. Chem. Res., Synop. 1999, 2, 136.

13. Iuhas, P. C.; Georgescu, F; Georgescu, E., Sci. Bull. Univ. POLITEHNICA Bucharest 1999, $B, 61(3-4), 85$. 